\title{
Control of ground-borne underground railway-induced vibration from double-deck tunnel infrastructures by means of dynamic vibration absorbers
}

\author{
Behshad Noori ${ }^{\mathrm{a}, *}$, Robert $\operatorname{Arcos}^{\mathrm{a}, \mathrm{c}}$, Arnau Clot ${ }^{\mathrm{b}}$, Jordi Romeu $^{\mathrm{a}}$ \\ ${ }^{a}$ Acoustical and Mechanical Engineering Laboratory (LEAM), Universitat Politècnica de \\ Catalunya $(U P C)$, Spain \\ ${ }^{b}$ Department of Engineering, Cambridge University, United Kingdom \\ ${ }^{c}$ Serra Húnter Fellow, Universitat Politècnica de Catalunya (UPC), Spain
}

\begin{abstract}
The aim of this study is to investigate the efficiency of Dynamic Vibration Absorbers (DVAs) as a vibration abatement solution for railway-induced vibrations in the framework of a double-deck circular railway tunnel infrastructure. A previously developed semi-analytical model of the track-tunnel-ground system is employed to calculate the energy flow resulting from a train pass-by. A methodology for the coupling of a set of longitudinal distributions of DVAs over a railway system is presented as a general approach, as well as its specific application for the case of the double-deck tunnel model. In the basis of this model, a Genetic Algorithm (GA) is used to obtain the optimal parameters of the DVAs to minimize the vibration energy flow radiated upwards by the tunnel. The parameters of the DVAs set to be optimized are the natural frequency, the viscous damping and their positions. The results show that the DVAs would be an effective countermeasure to address railway induced ground-borne vibration as the total energy flow radiated upwards from the tunnel can be reduced by an amount between $5.3 \mathrm{~dB}$ and $6.6 \mathrm{~dB}$ with optimized DVAs depending on the type of the soil and the train speed.
\end{abstract}

Keywords: Railway-induced vibration, Dynamic vibration absorber,

\footnotetext{
* Corresponding author

Email address: behshad.noori@upc.edu (Behshad Noori)
} 
Double-deck tunnel, Vibration energy flow

\section{Introduction}

The passage of trains in underground tunnels is one of the major sources of ground-borne vibrations. These vibrations propagate through the soil and structures of nearby buildings, resulting in undesired vibration and re-radiated noise inside the buildings. They may cause discomfort to the building residents, affect the operation of sensitive equipment and damage historical edifices with structural weakness. In recent years, innovative tunnel structure designs, like double-deck tunnels, have been constructed in several cities worldwide while few studies have been reported on appropriate measures to mitigate ground-borne vibration for these new designs.

Several countermeasures have been proposed to address the problem of ground-borne vibration induced by railways. These solutions can be categorized according to the location where they are applied: i) the source [1, 2]; ii) the receiver $[3,4]$ and iii) the propagation path $[5,6]$. Accurate prediction models should be used to assess the efficiency of these mitigation measures. Numerical, hybrid models and semi-analytical models can provide the desired level of accuracy. In the framework of numerical models, two-and-a-half-dimensional (2.5D) approaches based on Finite Element-Boundary Element Methods (FEM-BEM) [7-9], and the Method of Fundamental Solutions (MFS) coupled with FEM [10] are the common approaches. For specific sites, hybrid models [11, 12] can provide an increment on the accuracy with respect to conventional numerical approaches. Regarding semi-analytical models, probably the most well-established models for underground railway traffic is the Pipe-in-Pipe (PiP) model $[13,14]$. An extension of the PiP model was also presented by Hussein et al. [15] to calculate the vibrations from a tunnel embedded in a layered half-space, in which 2.5D Green's functions of a half-space can be evaluated using 3D stiffness matrices cast in cylindrical coordinates [16] or Cartesian coordinates [17].

A well-established system that has been widely used to control the vibration 
of mechanical, civil and aerospace structures is the Dynamic Vibration Absorber (DVA), also known as Tuned Mass Damper (TMD). In the last century, the application of DVAs as passive, active and semi-active countermeasures to attenuate undesirable vibration has been studied extensively $[18,19]$. Some of the prominent applications of DVAs around the globe are the ones in Taipei World Financial Center (also known as Taipei 101) [20], Millennium Bridge [21] and Doha Sport City Tower [22]. DVA is usually modeled as a single-degreeof-freedom (SDOF) and it works as a secondary oscillatory system applied on a primary system where the vibration needs to be controlled. The concept of a DVA was outlined by Watts in 1883 [23]. However, the practical design of a DVA, as a spring-supported mass, was proposed by Frahm in 1911 [24]. A damping element was later introduced to DVAs to widen the controlled frequency range [25]. DVAs can be used to attenuate the vibration at a specific frequency or over a particular range of frequencies. In the former case, the natural frequency of the DVA should be tuned to the specific frequency, and the damping should be chosen as low as possible [26]. In the latter case, an optimization criterion is required as the effectiveness of a DVA depends on its parameters.

Several types of optimization procedures with analytical or numerical approaches can be considered to determine the optimal value of DVA parameters [18, 27-29]. In most of the analytical optimization procedures that are employed to find the optimal value of DVA parameters, the structure on which the DVAs are applied (the host structure) is modeled as a SDOF system [28]. In these methodologies, the parameters of this SDOF model are obtained from the dominant mode of the host structure response. However, there are other optimization procedures that dont require a prior determination of which mode must be controlled. Among those, Genetic Algorithms (GAs) have been widely used for tuning DVA parameters in order to optimally reduce the vibration in specific locations of the system or in a global point of view. Some of the studies that apply GA for this purpose are [28, 30-33].

Recently, DVAs have been used to address some issues in the railway-induced 
vibration field. A study on the effectiveness of DVAs in suppressing the lowfrequency vibrations of floating slab track with discontinuous slabs was conducted by Zhu et al. [34] using a FEM model. They used two DVAs to minimize first- and second-mode vibrations of a slab which was treated as a SDOF system with the parameters of the selected mode, and the optimal parameters of DVAs were found using fixed-point theory [35]. Reducing the vibration of car-bodies of a low-floor train at a certain frequency by means of a DVA was investigated by Wang et al. [36], in which the DVA was found to be an effective countermeasure for excessive vertical vibration of car-bodies. DVAs have been also found to be effective in reducing the noise radiated by a rail. Thompson et al. [37] designed DVAs of steel masses and elastomeric material with a high damping loss factor, placed at both sides of the rail. The system of absorbers results in a reduction of radiated noise by about 5-6 dB. These DVAs were reported to be effective in slowing the growth of rail corrugation if they can fully suppress the pinnedpinned resonance [38]. Ho et al. developed multiple DVAs each consisting of a mass sandwiched between resilient materials [39]. This multiple mass-spring system has been put into practice and the system is found to be effective not only in attenuating rail vibration and the tunnel noise level [39] but also in slowing the growth of rail corrugation [40].

Double-deck circular tunnels are an innovative design underground transport infrastructures in which the tunnel is divided into two sections by an interior floor. The definition and design methodology of specific mitigation measures for ground-borne vibrations have not been studied adequately yet for this type of tunnel. The modification of the stiffness/damping values of the rail pads [41] and implementation of an elastomeric mat between the interior floor and the tunnel structure [41, 42] are two mitigation measures studied by Clot et al., using a $2.5 \mathrm{D}$ semi-analytical model of a double-deck tunnel [43]. It was found that implementing a soft elastomeric mat reduces the soil vibration considerably.

The potential of DVA as a vibration countermeasure for underground railwayinduced ground-borne vibration problems can be investigated for the full- and half-space cases as a model of the soil. The full-space model is used to generally 
evaluate the effect of DVAs by controlling the vibration energy radiated upwards by the tunnel structure. This model would be useful to address vibration problems of long railway sections, where different buildings and tunnel depths are involved, without of requiring a complicated model in which the particular foundation/geometry of nearby buildings need to be taken into account. On the other hand, the half-space model would be more convenient to study the vibration mitigation for specific buildings using a model in which the soil-building interaction is also considered.

In this paper, the application of DVAs on the upper section of a double-deck tunnel to minimize the energy flow radiated upwards resulting from the train pass-by over this section is studied through the full-space approach. The vehicletrack-tunnel-soil model is employed, in which the track-tunnel-soil model is the one developed by Clot et al. [43] and the train-track model is an extension of the model presented in [44] by considering two $2 \mathrm{D}$ vehicle models over the two rails. The rest of the paper is organized as follows: In Section 2, the vehicle-track-tunnel-soil model is described; Section 3 is dedicated to details regarding the coupling of DVAs to an underground railway infrastructure model. The optimization method to determine the optimal parameters of the DVAs to minimize energy flow radiated upwards for the case of an underground doubledeck tunnel railway infrastructure is presented in Section 4. The efficiency of a set of DVAs as a vibration countermeasure applied to the specific case studies to minimize the radiated energy flow is discussed in Section 5. Finally, results obtained from this study are summed up in Section 6 .

\section{Modeling of the vehicle-track-tunnel-soil system}

This section starts by recapitulating the considered 2.5D semi-analytical model of a double-deck tunnel embedded in a full-space [43]. This model provides the 2.5D Green's functions of the system due to loads applied on the rails and on the interior floor, which are required to couple the train and the DVAs to the system and to compute the train pass-by response. Then, the considered 
train-track interaction model is described, followed by an explanation of how this model is employed to determine the response of the system due to a train pass-by.

\subsection{Track-tunnel-soil model}

A scheme of the model considered for the track-tunnel-soil system is shown in Fig. 1. The track consists of two rails connected to the interior floor of the double-deck tunnel structure by means of direct fixation fasteners, and the tunnel is assumed to be embedded in a homogeneous full-space. The system is considered to be invariant in the longitudinal direction, i.e., the direction in which the train circulates.

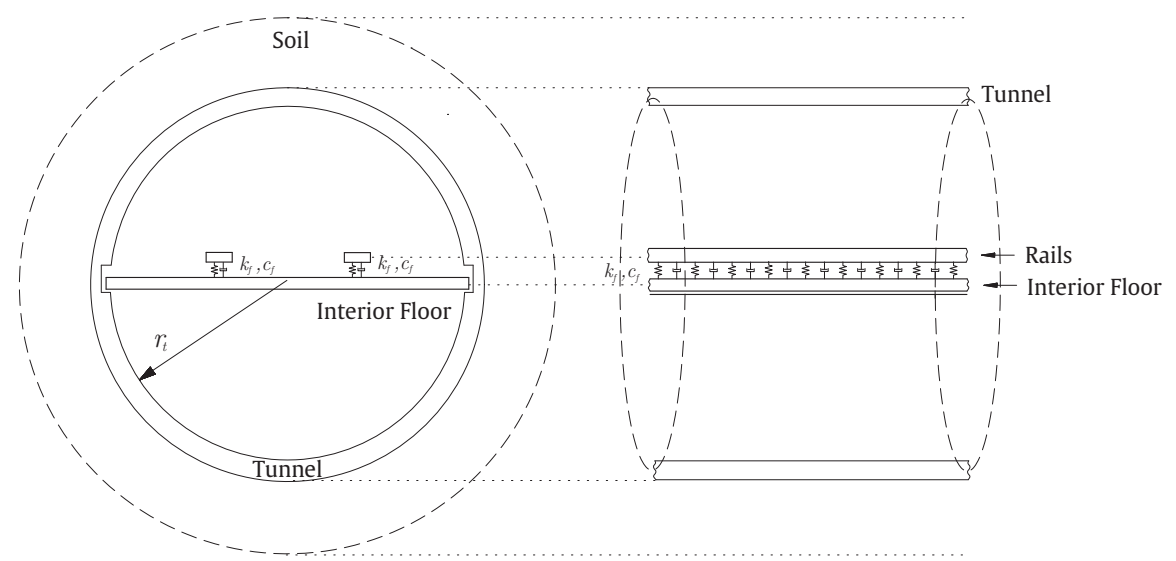

Fig. 1: A scheme of a double-deck tunnel and its subsystems embedded in a full-space.

The rails are modeled as Euler-Bernoulli beams of infinite length separated by a specific distance. The direct fixation fastener is represented by a continuous mass-less distribution of springs, with a stiffness per unit length $k_{f}$, and dashpots, with a viscous damping per meter $c_{f}$. The interior floor is modeled as a homogeneous isotropic thin strip plate with a rectangular cross-section. The tunnel and soil subsystems are described using the PiP model, developed by Forrest and Hunt [13], which assumes that they can be represented as an infinite thin cylindrical shell and as an infinite homogeneous elastic medium, 
respectively.

\subsection{Vehicle-track coupling}

There are two excitation mechanisms that mostly contribute to the vibration induced by railway traffic: i) the quasi-static excitation ii) the dynamic excitation. The former is induced by the static component of the moving loads applied by the train to the track and is of great importance for high-speed trains. The latter can be attributed to various mechanisms [45], mainly the wheel/rail unevenness and the longitudinal variability of the track's mechanical parameters. Since the present investigation is done in the context of urban railway infrastructure, the vertical dynamic excitation caused by the rail unevenness is considered as the only excitation source. It is assumed that the unevenness of the rails is uncorrelated between them [46].

Consider a moving frame of reference associated to a train motion. Due to the Doppler effect, the frequency components of the time signals seen from the perspective of this moving frame of reference $(\tilde{\omega})$ are different from the ones related to a fixed frame of reference $(\omega)$ [44]. All the derivation presented in this section is based on the moving coordinate system, thus, all the variables represented in the frequency domain are associated to the frequency $\tilde{\omega}$, except when it is specifically mentioned otherwise. Capital letters notation is used to denote variable in the frequency domain.

The vertical displacements of the two rails in the frequency domain due to the wheel/rail contact forces can be represented by

$$
\left\{\begin{array}{l}
\mathbf{Z}_{r_{1}}^{w / r} \\
\mathbf{Z}_{r_{2}}^{w / r}
\end{array}\right\}=\left\{\begin{array}{ll}
\mathbf{H}_{r_{1} r_{1}}^{w / r} & \mathbf{H}_{r_{1} r_{2}}^{w / r} \\
\mathbf{H}_{r_{2} r_{1}}^{w / r} & \mathbf{H}_{r_{2} r_{2}}^{w / r}
\end{array}\right\}\left\{\begin{array}{l}
\mathbf{F}_{r_{1}}^{w / r} \\
\mathbf{F}_{r_{2}}^{w / r}
\end{array}\right\},
$$

where $\mathbf{Z}_{r_{1}}^{w / r}$ and $\mathbf{Z}_{r_{2}}^{w / r}$ are the vertical displacements of the left and right rails, respectively, at all the vehicle axle positions, $\mathbf{H}_{r_{1} r_{1}}^{w / r}$ and $\mathbf{H}_{r_{2} r_{2}}^{w / r}$ are the direct receptance matrices of the left and right rails, respectively, at all the axles positions, $\mathbf{H}_{r_{2} r_{1}}^{w / r}=\mathbf{H}_{r_{1} r_{2}}^{w / r}$ is the cross receptance matrix between the left and 
right rails at all axle positions, $\mathbf{F}_{r_{1}}^{w / r}$ and $\mathbf{F}_{r_{2}}^{w / r}$ are the vectors of wheel/rail interaction forces associated to the left and right rails, respectively; and the response of the two half vehicles can be written by

$$
\left\{\begin{array}{l}
\mathbf{Z}_{v_{1}}^{w / r} \\
\mathbf{Z}_{v_{2}}^{w / r}
\end{array}\right\}=-\left\{\begin{array}{cc}
\mathbf{H}_{v_{1}}^{w / r} & 0 \\
0 & \mathbf{H}_{v_{2}}^{w / r}
\end{array}\right\}\left\{\begin{array}{l}
\mathbf{F}_{r_{1}}^{w / r} \\
\mathbf{F}_{r_{2}}^{w / r}
\end{array}\right\},
$$

where $\mathbf{Z}_{v_{1}}^{w / r}$ and $\mathbf{Z}_{v_{2}}^{w / r}$ are the vertical displacements of vehicle wheels in contact with the left and right rails, respectively, and $\mathbf{H}_{v_{1}}^{w / r}$ and $\mathbf{H}_{v_{2}}^{w / r}$ are the receptances of each half vehicle at all the vehicle axle positions. Eqs. (1) and (2) can easily be compacted to

$$
\mathbf{Z}_{r}^{w / r}=\mathbf{H}_{r}^{w / r} \mathbf{F}^{w / r}, \quad \mathbf{Z}_{v}^{w / r}=-\mathbf{H}_{v}^{w / r} \mathbf{F}^{w / r}
$$

Consider now that the 2.5D Green's functions associated to the studied railway infrastructure system are obtained on the basis of a double Fourier transform (FT) defined by

$$
\bar{G}\left(k_{x}, \omega\right)=\int_{-\infty}^{+\infty} \int_{-\infty}^{+\infty} g(x, t) \mathrm{e}^{\mathrm{i}\left(k_{x} x-\omega t\right)} \mathrm{d} x \mathrm{~d} t
$$

where $x, t, k_{x}$ and $\omega$ represent the longitudinal coordinate, the time, the wavenumber associated to the longitudinal coordinate and the frequency seen from a fixed frame of reference, respectively. Combined bar and capital letters notation is used to denote variables in the wavenumber-frequency domain on a fixed frame of reference. Taking this into account, the elements of the receptance matrices required to construct $\mathbf{H}_{r}^{w / r}$ can be computed by

$$
H_{r_{i} r_{j}, n m}^{w / r}=\frac{1}{2 \pi} \int_{-\infty}^{+\infty} \tilde{H}_{r_{i} r_{j}} \mathrm{e}^{-\mathrm{i} k_{x}\left(\tilde{x}_{n}-\tilde{x}_{m}\right)} \mathrm{d} k_{x}
$$

where $H_{r_{i} r_{j}, n m}^{w / r}$ is the $(n, m)$ element of the matrix $\mathbf{H}_{r_{i} r_{j}}^{w / r}, \tilde{x}_{n}$ and $\tilde{x}_{m}$ are the longitudinal coordinates of the $n$-th and $m$-th axles, respectively, seen from the point of view of the moving frame of reference, and $\tilde{H}_{r_{i} r_{j}}$ is the $2.5 \mathrm{D}$ Green's 
function of the system defined in the $\left(k_{x}, \tilde{\omega}\right)$ domain that relates the vertical motions of the rails $r_{i}$ and $r_{j}$. A $2.5 \mathrm{D}$ Green's function defined in the $\left(k_{x}, \tilde{\omega}\right)$ domain can be obtained from the one defined in the $\left(k_{x}, \omega\right)$ domain by $\tilde{H}\left(k_{x}, \tilde{\omega}\right)=\bar{H}\left(k_{x}, \tilde{\omega}+k_{x} v_{t}\right)$, being $v_{t}$ the speed of the train. Note that combined $\sim$ and capital letters notation is used to denote variables in the wavenumberfrequency domain on a moving frame of reference. The vehicle receptance matrix is obtained by means of the dynamic model of the vehicle, which in this investigation is considered to be the two-dimensional (2D) multi-degree-of-freedom rigid body model presented by Lei and Noda [47]. A 3D model of each car consists of two uncoupled 2D models separately applied on each rail. A global train is modeled as a set of $N_{c}$ identical cars.

Assuming a linearized Hertz contact, the wheel/rail interaction forces can be obtained in the frequency domain by using

$$
\mathbf{F}^{w / r}=k_{H}\left(\mathbf{Z}_{v}^{w / r}-\mathbf{Z}_{r}^{w / r}+\mathbf{E}_{r}\right)
$$

where $k_{H}$ is the stiffness of the linearized Hertzian spring, considered to be the same in all the wheel/rail contacts, and $\mathbf{E}_{r}$ is the vector of complex amplitudes of rails unevenness at all the wheel/rail contacts. Combining Eq. (6) with Eq. (3) one can obtain a transfer function in the frequency domain between the unevenness of the rails and the dynamic wheel/rail interaction forces, which may be written as

$$
\mathbf{F}^{w / r}=\left(\mathbf{H}_{v}^{w / r}+\mathbf{H}_{r}^{w / r}+k_{H}^{-1} \mathbf{I}\right)^{-1} \mathbf{E}_{r}
$$

where $\mathbf{I}$ is the identity matrix.

Once the wheel/rail interaction forces are computed, the response at an arbitrary position $l$ of the railway infrastructure system due to the passage of the train can be found using the expression

$$
u_{l}(\tilde{x}, t)=\sum_{i=1}^{2} \frac{1}{2 \pi} \int_{-\infty}^{+\infty} \sum_{n=1}^{N_{a}}\left[\frac{1}{2 \pi} \int_{-\infty}^{+\infty} \tilde{H}_{l r_{i}} F_{r_{i}, n}^{w / r} \mathrm{e}^{-\mathrm{i} k_{x}\left(\tilde{x}-\tilde{x}_{n}\right)} \mathrm{d} k_{x}\right] \mathrm{e}^{\mathrm{i} \tilde{\omega} t} \mathrm{~d} \tilde{\omega}
$$


where $u_{l}(\tilde{x}, t)$ is the displacement response at $l$ position of the railway infrastructure system, $\tilde{H}_{l r_{i}}$ is the $2.5 \mathrm{D}$ Green's function in the wavenumber-frequency domain that relates the displacement response at that arbitrary position $l$ with a force applied in the $i$-th rail, $\tilde{x}$ is the longitudinal coordinate associated to the moving frame of reference, $N_{a}$ is the number of axles of the train and $F_{r_{i}, n}^{w / r}$ is the wheel/rail interaction force associated to the $i$-th rail and the $n$-th axle. An equivalent expression for the soil tractions can be obtained by simply replacing the displacement Green's functions with those for the tractions in Eq. (8). The expression of the soil vibration velocity can be written similarly to Eq. (8) as $v_{l}(\tilde{x}, t)=\sum_{i=1}^{2} \frac{1}{2 \pi} \int_{-\infty}^{+\infty} \sum_{n=1}^{N_{a}}\left[\frac{1}{2 \pi} \int_{-\infty}^{+\infty} \mathrm{i}\left(\tilde{\omega}+k_{x} v_{t}\right) \tilde{H}_{l r_{i}} F_{r_{i}, n}^{w / r} \mathrm{e}^{-\mathrm{i} k_{x}\left(\tilde{x}-\tilde{x}_{n}\right)} \mathrm{d} k_{x}\right] \mathrm{e}^{\mathrm{i} \tilde{\omega} t} \mathrm{~d} \tilde{\omega}$,

\section{Application of DVAs on an underground railway system}

This section starts with the explanation of a methodology which can be used to couple a set of DVAs to any subsystem of a railway infrastructure. Then, the application of this methodology for the case of the double-deck tunnel is explained.

DVAs can be applied on different subsystems of a railway infrastructure, such as the tunnel or the track. For example, Fig. 2 shows a cross-section of a track with one longitudinal distribution of DVAs. Consider $M$ longitudinal distributions of DVAs, where each distribution has $N_{m}$ DVAs, being $m=1,2, \ldots, M$. The total amount of DVAs, then, is $\sum_{m=1}^{M} N_{m}$. In the following the $n$-th DVA of the $m$-th distribution is represented by $d_{m n}$.

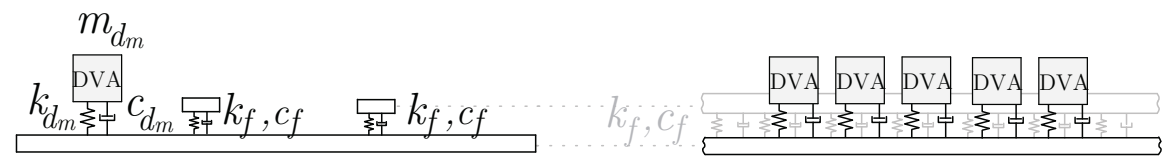

Fig. 2: A track system with one longitudinal distribution of DVAs.

Considering each DVA as a SDOF system, as shown in Fig. 3, the equation 
of motion of the $n$-th DVA of the $m$-th distribution can be written as

$$
-c_{d_{m n}}\left(\dot{z}_{d_{m n}}^{c}-\dot{z}_{d_{m n}}\right)-k_{d_{m n}}\left(z_{d_{m n}}^{c}-z_{d_{m n}}\right)=m_{d_{m n}} \ddot{z}_{d_{m n}},
$$

where $z_{d_{m n}}^{c}$ and $z_{d_{m n}}$ are the displacement of the DVA and the displacement of the system at the position of the DVA, respectively, in a direction perpendicular to the surface on which DVAs are applied. $m_{d_{m n}}, k_{d_{m n}}$ and $c_{d_{m n}}$ are the mass, stiffness and viscous damping coefficient of $d_{m n}$.

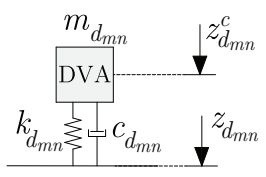

Fig. 3: DVAs modeled as a SDOF.

Using a FT over time domain, based on the definition presented in Eq. (4), the equation of motion can be transformed to the frequency domain

$$
-\omega^{2} m_{d_{m n}} Z_{d_{m n}}+\mathrm{i} \omega c_{d_{m n}}\left(Z_{d_{m n}}^{c}-Z_{d_{m n}}\right)+k_{d_{m n}}\left(Z_{d_{m n}}^{c}-Z_{d_{m n}}\right)=0 .
$$

Then, assuming that all DVAs of the $m$-th distribution have the same mass $m_{d_{m}}$, stiffness $k_{d_{m}}$ and damping coefficient $c_{d_{m}}$, the relation between the displacement of the DVA $Z_{d_{m n}}^{c}$ and the displacement of the system at the position of the DVA $Z_{d_{m n}}$ can be found as

$$
Z_{d_{m n}}^{c}=\frac{k_{d_{m}}+\mathrm{i} \omega c_{d_{m}}}{-m_{d_{m}} \omega^{2}+k_{d_{m}}+\mathrm{i} \omega c_{d_{m}}} Z_{d_{m n}}
$$

The forces applied to the system due to the DVAs of the $m$-th distribution can be written as

$$
f_{d_{m}}(x, t)=\sum_{n=1}^{N_{m}}\left(k_{d_{m}}+c_{d_{m}} \frac{\partial}{\partial t}\right) \delta\left(x-x_{d_{m n}}\right)\left(z_{d_{m n}}^{c}-z_{d_{m n}}\right)
$$

where $x_{d_{m n}}$ is the position of $d_{m n}$ in the longitudinal direction. These forces can 
be transformed to the wavenumber-frequency domain using Eq. (4), resulting in

$$
\bar{F}_{d_{m}}\left(k_{x}, \omega\right)=\sum_{n=1}^{N_{m}}\left(k_{d_{m}}+\mathrm{i} \omega c_{d_{m}}\right) \mathrm{e}^{\mathrm{i} k_{x} x_{d_{m n}}}\left(Z_{d_{m n}}^{c}-Z_{d_{m n}}\right) .
$$

Finally, by introducing Eq. (12) into Eq. (14), the forces applied to the interior floor by the DVAs can be rewritten as

$$
\bar{F}_{d_{m}}\left(k_{x}, \omega\right)=\sum_{n=1}^{N_{m}} k_{d_{m}}^{*} \mathrm{e}^{\mathrm{i} k_{x} x_{d_{m n}}} Z_{d_{m n}}
$$

where

$$
k_{d_{m}}^{*}=\frac{\omega^{2} m_{d_{m}}\left(k_{d_{m}}+\mathrm{i} \omega c_{d_{m}}\right)}{-\omega^{2} m_{d_{m}}+\mathrm{i} \omega c_{d_{m}}+k_{d_{m}}} .
$$

Therefore, the forces applied to the system by all $M$ distributions of DVAs can be written in matrix form as

$$
\overline{\mathbf{F}}_{d}=\boldsymbol{\Gamma}_{d} \mathbf{K}_{d}^{*} \mathbf{Z}_{d}
$$

where

$$
\overline{\mathbf{F}}_{d}=\left\{\begin{array}{c}
\bar{F}_{d_{1}} \\
\bar{F}_{d_{2}} \\
\vdots \\
\bar{F}_{d_{m}} \\
\vdots \\
\bar{F}_{d_{M}}
\end{array}\right\}, \quad \mathbf{Z}_{d}=\left\{\begin{array}{c}
\mathbf{z}_{d_{1}} \\
\mathbf{z}_{d_{2}} \\
\vdots \\
\mathbf{z}_{d_{m}} \\
\vdots \\
\mathbf{z}_{d_{M}}
\end{array}\right\},
$$

where $\mathbf{Z}_{d_{m}}$ is a vector which contains the displacements of the system at the positions of all the DVAs of the $m$-th distribution. The other matrices are 
defined as

$$
\mathbf{K}_{d}^{*}=\left[\begin{array}{cccccc}
\mathbf{K}_{d_{1}}^{*} & & & & & \\
& \mathbf{K}_{d_{2}}^{*} & & & & \\
& & \ddots & & & \\
& & & \mathbf{K}_{d_{m}}^{*} & & \\
& & & & \ddots & \\
& & & & & \mathbf{K}_{d_{M}}^{*}
\end{array}\right],
$$

where $\mathbf{K}_{d_{m}}^{*}$ is a $N_{m} \times N_{m}$ matrix defined by $k_{d_{m}}^{*} \mathbf{I}$; and

$$
\boldsymbol{\Gamma}_{d}=\left[\begin{array}{cccccc}
\boldsymbol{\Gamma}_{d_{1}} & & & & & \\
& \boldsymbol{\Gamma}_{d_{2}} & & & & \\
& & \ddots & & & \\
& & & \boldsymbol{\Gamma}_{d_{m}} & & \\
& & & \ddots & \\
& & & & & \boldsymbol{\Gamma}_{d_{M}}
\end{array}\right],
$$

where

$$
\boldsymbol{\Gamma}_{d_{m}}=\left\{\begin{array}{llll}
\mathrm{e}^{\mathrm{i} k_{x} x_{d_{m 1}}} & \mathrm{e}^{\mathrm{i} k_{x} x_{d_{m 2}}} & \cdots & \mathrm{e}^{\mathrm{i} k_{x} x_{d_{m N_{m}}}}
\end{array}\right\}
$$

In the following, it is assumed that the force is applied only on one of the rails. For the case of the two forces applied on the two rails, linear superposition can be held. Moreover, the following derivation is based on the moving frame of reference, as explained in Section 2. All the variables represented in the frequency domain in this section are thus associated to the frequency $\tilde{\omega}$. The system's response at the position of the DVAs can be obtained from

$$
\tilde{\mathbf{Z}}_{d}=\tilde{\mathbf{H}}_{d r} \tilde{F}_{r}+\tilde{\mathbf{H}}_{d d} \tilde{\mathbf{F}}_{d}
$$

where $\tilde{\mathbf{H}}_{d r}$ refers to the 2.5D Green's function for the displacement of the system at the DVAs positions due to a force on the rail seen in the moving frame of reference in the absence of the DVAs; $\tilde{F}_{r}$ is a force applied on the rails in the 
moving frame of reference; and $\tilde{\mathbf{H}}_{d d}$ refers to the 2.5D Green's function for displacements of the system at the DVAs positions due to a force applied on the DVAs positions. Replacing $\tilde{\mathbf{F}}_{d}$ with its equivalent from Eq. (17), Eq. (22) can be rewritten in the form of 2.5D Green's functions as

$$
\tilde{\mathbf{H}}_{d r}^{d}=\tilde{\mathbf{H}}_{d r}+\tilde{\mathbf{H}}_{d d} \boldsymbol{\Gamma}_{d} \tilde{\mathbf{K}}_{d}^{*} \mathbf{H}_{d r}^{d}
$$

where $\tilde{\mathbf{H}}_{d r}^{d}$ is the 2.5D Green's function that relates the displacement in the DVAs positions with a force in the rails seen in the moving frame of reference in the presence of the DVAs and $\mathbf{H}_{d r}^{d}$ is its inverse FT over the defined wavenumber by using the same structure as $\mathbf{Z}_{d}$ in Eq. (18). Transforming Eq. (23) to the space-frequency domain by applying an inverse FT over the wavenumber and evaluating the transformed equation at the positions of the DVAs one can obtain the expression

$$
\mathbf{H}_{d r}^{d}=\mathbf{H}_{d r}+\mathbf{H}_{d d} \mathbf{K}_{d}^{*} \mathbf{H}_{d r}^{d}
$$

where

$$
\mathbf{H}_{d r}=\left\{\begin{array}{c}
\mathbf{H}_{d_{1} r} \\
\mathbf{H}_{d_{2} r} \\
\vdots \\
\mathbf{H}_{d_{m} r} \\
\vdots \\
\mathbf{H}_{d_{M} r}
\end{array}\right\},
$$

being $\mathbf{H}_{d_{m} r}$ the receptances of the system at the DVAs positions of $m$-th distribution due to the force applied on one of the rails, defined as

$$
\mathbf{H}_{d_{m} r}=\frac{1}{v_{t}} \overline{\mathbf{H}}_{d_{m} r} \boldsymbol{\Gamma}_{d_{m}}^{\mathrm{T}}
$$


and where

$$
\mathbf{H}_{d d}=\left[\begin{array}{cccccc}
\mathbf{H}_{d_{1} d_{1}} & \mathbf{H}_{d_{1} d_{2}} & \ldots & \mathbf{H}_{d_{1} d_{p}} & \ldots & \mathbf{H}_{d_{1} d_{M}} \\
\mathbf{H}_{d_{2} d_{1}} & \mathbf{H}_{d_{2} d_{2}} & \ldots & \mathbf{H}_{d_{2} d_{p}} & \ldots & \mathbf{H}_{d_{2} d_{M}} \\
\vdots & \vdots & \ddots & \vdots & \ddots & \vdots \\
\mathbf{H}_{d_{m} d_{1}} & \mathbf{H}_{d_{m} d_{2}} & \ldots & \mathbf{H}_{d_{m} d_{p}} & \ldots & \mathbf{H}_{d_{m} d_{M}} \\
\vdots & \vdots & \ddots & \vdots & \ddots & \vdots \\
\mathbf{H}_{d_{M} d_{1}} & \mathbf{H}_{d_{M} d_{2}} & \ldots & \mathbf{H}_{d_{M} d_{p}} & \ldots & \mathbf{H}_{d_{M} d_{M}}
\end{array}\right]
$$

being $\mathbf{H}_{d_{m} d_{p}}$ a $N_{m} \times N_{p}$ matrix which contains receptance matrices of the system at the DVAs positions of the $m$-th distribution due to the forces applied on the system at the DVAs positions of the $p$-th distribution. Each element of these matrices can be determined by

$$
\begin{gathered}
H_{d_{m} d_{p}, j q}=\frac{1}{2 \pi} \int_{-\infty}^{+\infty} \tilde{H}_{d_{m} d_{p}, j q} \mathrm{e}^{\mathrm{i} k_{x}\left(x_{d_{m}}^{j}-x_{d_{p}}^{q}\right)} \mathrm{d} k_{x} \\
j=1,2, \ldots, N_{m}, \quad q=1,2, \ldots, N_{p}, k_{x} \kappa_{x}
\end{gathered}
$$

where $x_{d_{m}}^{j}$ is the position of $j$-th DVA in the $m$-th distribution in the longitudinal direction, and $x_{d_{p}}^{q}$ is the position of $q$-th DVA in the $p$-th distribution in the longitudinal direction.

Finally, operating Eq. (24), the receptance of the system at the DVAs positions in the presence of the DVAs can be obtained as

$$
\mathbf{H}_{d r}^{d}=\left(\mathbf{I}-\mathbf{H}_{d d} \mathbf{K}_{d}\right)^{-1} \mathbf{H}_{d r}
$$

Having these receptances, one can obtain the 2.5D Green's functions of the system at any arbitrary position $l$ due to the force applied at the rail in the presence of the DVAs as

$$
\tilde{\mathbf{H}}_{l r}^{d}=\tilde{\mathbf{H}}_{l r}+\tilde{\mathbf{H}}_{l d} \boldsymbol{\Gamma}_{d} \tilde{\mathbf{K}}_{d}^{*} \mathbf{H}_{d r}^{d}
$$

where the $\tilde{\mathbf{H}}_{l r}$ refers to the 2.5D Green's function of the system in the absence 
of the DVAs. It is noteworthy that this methodology considers a strong coupling approach, in which the DVAs affect the response of the rails.

In this paper, the application of the DVAs is studied in the context of a double-deck tunnel. Using the process explained previously, $M$ longitudinal distribution of DVAs can be coupled to the interior floor of this type of tunnels. Fig. 4 shows a cross-section of the double-deck tunnel model with one longitudinal distribution of DVAs, which is the model that will be used in the following sections.

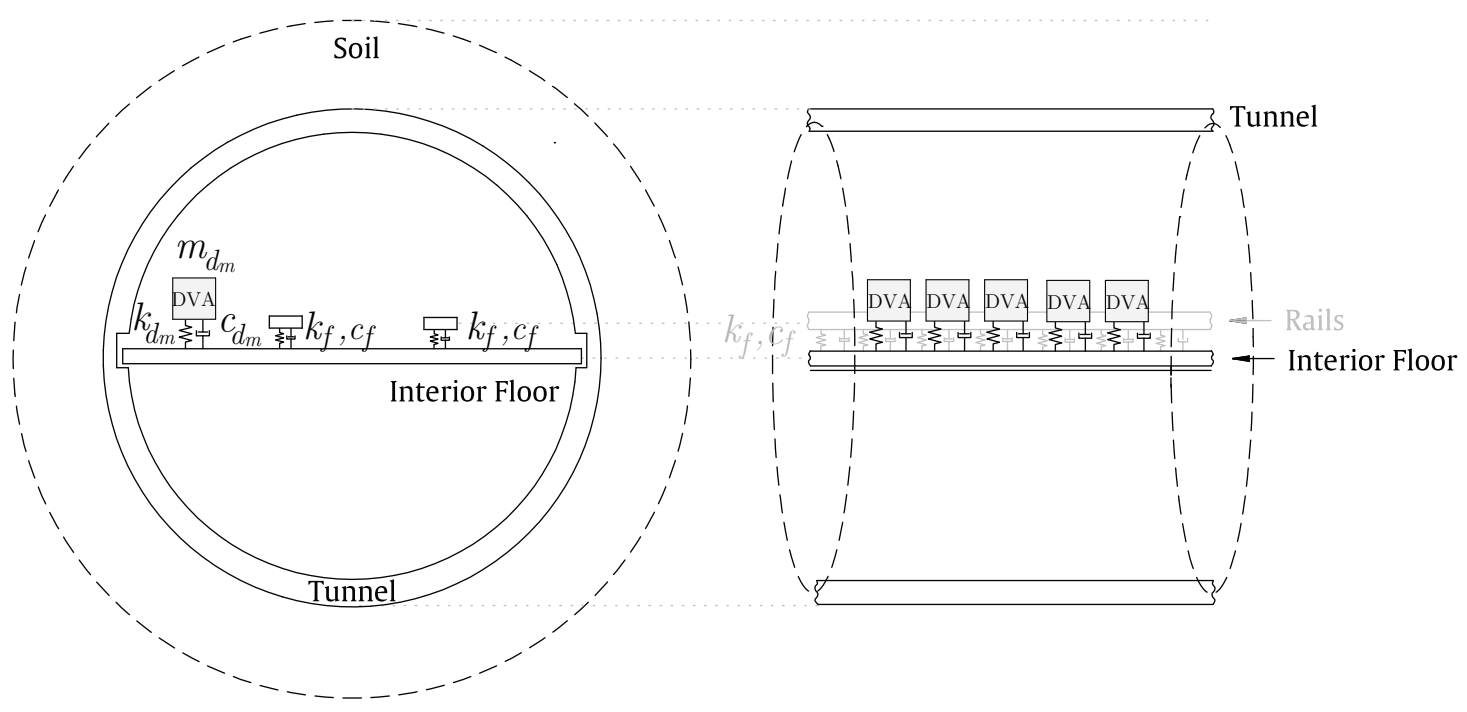

Fig. 4: The track-tunnel-soil model in full-space with one distribution of DVAs.

\section{DVAs optimization approach}

A global optimization approach based on GA is used in this paper to obtain the optimum parameters of DVAs as countermeasures for underground railwayinduced ground-borne vibration. Design variables that affect the performance of DVAs are: number of DVAs distributions, position of DVAs distributions, number of DVAs in each distribution, distance between two consecutive DVAs in each distribution, DVAs masses, DVAs natural frequencies and DVAs damping 
coefficients. In the optimization process, the effectiveness of DVAs is assessed by their performance in minimizing energy flow radiated upwards.

The mean power flow radiated upwards from a tunnel towards nearby buildings was proposed by Hussein and Hunt [48] as a criterion to evaluate the performance of vibration countermeasures. Studies of power flow and energy flow that radiate upwards from a double-deck tunnel are presented by Clot et al. [49] and [50], respectively. The radiated energy flow is the one used in this study to assess the efficiency of DVAs and, in the following, it is explained how to compute it.

For a double-deck tunnel, the vibration energy radiating upwards through a cylindrical strip (shown in Fig. 5) at any arbitrary cross section $x_{e}$ due to the passage of the train can be determined by integrating the power flow that crosses through the cylindrical strip as

$$
E=r_{m} \Delta \mathrm{x} \int_{\theta_{1}}^{\theta_{2}} \int_{-\infty}^{+\infty} \mathbf{v}\left(x_{e}, \theta, t\right) \cdot \boldsymbol{\tau}\left(x_{e}, \theta, t\right) \mathrm{d} t \mathrm{~d} \theta,
$$

where $\mathbf{v}$ and $\boldsymbol{\tau}$ are the soil vibration velocity and tractions, respectively, at the strip due to the train pass-by. Tractions here refer to the stresses projected to the cylindrical strip surface.

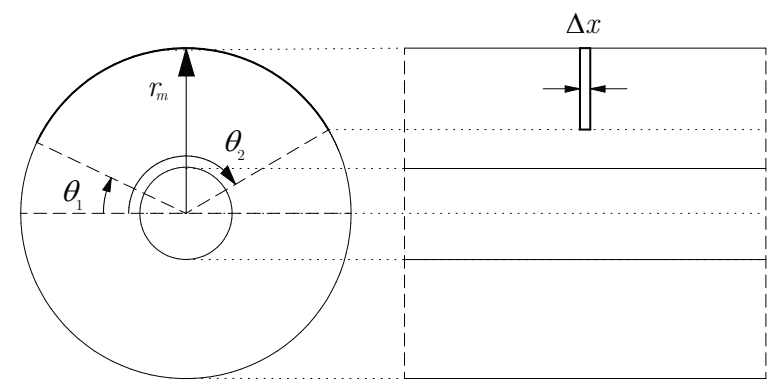

Fig. 5: A finite cylindrical strip through which the radiated energy flow is calculated.

To assess the performance of the DVAs by using the energy flow criterion, it is necessary to compute the vibration energy radiating upwards for the cases in which the DVAs are applied to the system and in which they are not. For the 
case without DVAs, the displacement and traction Green's functions that relates the response on the soil at the cylindrical strip due to a force applied on the $i$-th rail can be obtained using the model explained in Subsection 2.1. These Green's functions can be used to obtain the response on the soil at the cylindrical strip due a train pass-by by applying the formulation presented in Subsection 2.2 and, finally, to obtain the energy flow radiated upwards by the tunnel using Eq. (31). For the case in which the DVAs are coupled to the tunnel's interior floor, the same procedure can be followed using the Green's functions that accounts for the DVAs application, which can be found by following Section 3 .

\section{Application and results}

In this section, the efficiency of the application of the optimized DVAs on the interior floor of the double-deck tunnel in minimizing the energy flow radiated upwards by the tunnel is investigated. The considered mechanical properties for the different subsystems are described first in Subsection 5.1. Then, in Subsection 5.2, it is explained how the required Green's function have been computed, concerning the position of the receivers, possible position of DVAs and the wavenumber-frequency sampling. The train pass-by response is computed in Subsection 5.3. In Subsection 5.4, optimized parameters of DVAs to minimize energy flow radiated upwards are computed by using the previously explained optimization procedure, and the effects of the optimized DVAs are discussed.

\subsection{Parameters used to model subsystems}

Two types of soil are considered, soft soil and hard soil. Their mechanical parameters are summarized in Table 1. The purpose of using a low Youngs modulus for the soft soil case is to assess the performance of the DVAs in an extreme scenario. The mechanical and geometric parameters of the tunnel and the interior floor can be found in Tables 2 and 3, respectively. Typical values of reinforced concrete are used to model the tunnel and the interior floor. 
Table 1: Mechanical parameters used to model the soil.

Table 2: Mechanical parameters used to model the tunnel.

\begin{tabular}{cc}
\hline Tunnel parameters & Values \\
\hline Young modulus $(\mathrm{GPa})$ & 50 \\
Density $\left(\mathrm{kg} \mathrm{m}^{-3}\right)$ & 3000 \\
Poisson ratio $(-)$ & 0.175 \\
Thickness $(\mathrm{m})$ & 0.4 \\
Interior radius $(\mathrm{m})$ & 5.65 \\
\hline
\end{tabular}

Table 3: Mechanical parameters used to model the interior floor.

\begin{tabular}{cc}
\hline Interior floor parameters & Values \\
\hline Young modulus (GPa) & 30 \\
Density $\left(\mathrm{kg} \mathrm{m}^{-3}\right)$ & 3000 \\
Poisson ratio $(-)$ & 0.175 \\
Thickness $(\mathrm{m})$ & 0.5 \\
Width $(\mathrm{m})$ & 10.9
\end{tabular}

The track consists of two identical rails separated at a constant distance of $1.5 \mathrm{~m}$ and a continuous mass-less distribution of springs-dashpots as a model of the fasteners. Their parameters are given in Tables 4 and 5 . 
Table 4: Mechanical parameters used to model the rail.

\begin{tabular}{cc}
\hline Rail parameters & Values \\
\hline Young modulus $(\mathrm{GPa})$ & 207 \\
Density $\left(\mathrm{kg} \mathrm{m}^{-3}\right)$ & 7850 \\
Cross-section area $\left(\mathrm{m}^{2}\right)$ & $6.93 \cdot 10^{-3}$ \\
Second moment of area $\left(\mathrm{m}^{4}\right)$ & $23.5 \cdot 10^{-6}$ \\
\hline
\end{tabular}

Table 5: Mechanical parameters used to model the fastener.

\begin{tabular}{cc}
\hline Fasteners parameters & Values \\
\hline Uniformly distributed stiffness $\left(\mathrm{N} \mathrm{m}^{-2}\right)$ & $20 \cdot 10^{6}$ \\
Uniformly distributed viscous damping $\left(\mathrm{N} \mathrm{s} \mathrm{m}^{-2}\right)$ & $10 \cdot 10^{3}$ \\
\hline
\end{tabular}

The considered train consists of two identical 3D models of the vehicle, shown in Fig. 6. The distance between the wheels of a bogie, bogies of a same car and bogies of two consecutive cars are $2.2 \mathrm{~m}, 15 \mathrm{~m}$ and $7 \mathrm{~m}$, respectively. The parameters of the $2 \mathrm{D}$ vehicle models referred to in Subsection 2.2 are: $m_{w}$ represents the mass of the combined wheel and $1 / 2$-axle system; $m_{\mathrm{bog}}$ and $J_{\mathrm{bog}}$ represent the mass and mass of inertia of a $1 / 2$-bogie, respectively; $k_{p s}$ and $c_{p s}$ represent the stiffness and viscous damping, respectively, of the primary vehicle suspension system; $m_{\mathrm{c}}$ and $J_{\mathrm{c}}$ represent the mass and mass of inertia of a $1 / 2$-car body; and $k_{s s}$ and $c_{s s}$ represent the stiffness and viscous damping, respectively, of the secondary vehicle suspension system. The values for these parameters can be found in Table 6.

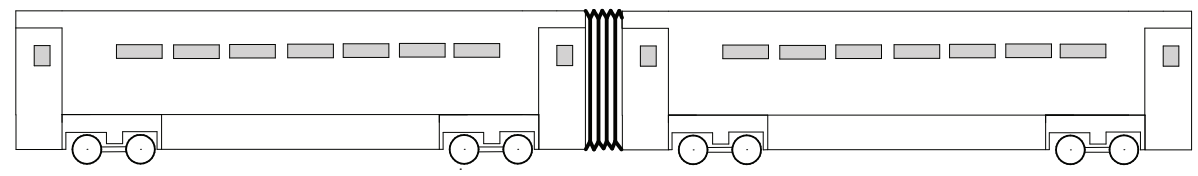

Fig. 6: Train configuration. 
Table 6: Mechanical parameters used to model the train.

\subsection{Computation of the Green's functions}

The track-tunnel-soil model presented in Subsection 2.1, along with the parameters of the subsystems given in the previous section, is used to compute the Green's functions required for coupling the vehicle and DVAs to the track and the interior floor, respectively, and for computing the energy flow radiated upwards due to the passage of the train. The computation of the energy flow radiated upwards takes into account a set of receivers on the soil at a semicircle concentric with the tunnel and with the radius $r_{s}=12 \mathrm{~m}$. A total amount of 21 receivers with an angular resolution of $9 \pi / 20 \mathrm{rad}$ are spread out across the semicircle. For the coupling between the DVAs and the interior floor, 20 receivers along the $y$ coordinate over the interior floor are considered. They are the possible DVAs positions that are considered in the optimization process. These receivers are equidistantly spread out across the interior floor considering a space resolution of $0.5 \mathrm{~m}$, and a distance of $0.5 \mathrm{~m}$ from each edge of the interior floor. The receivers in the soil and the interior floor, denoted by $d_{m}$ and $s_{l}$, respectively, are shown in Fig. 7 . 


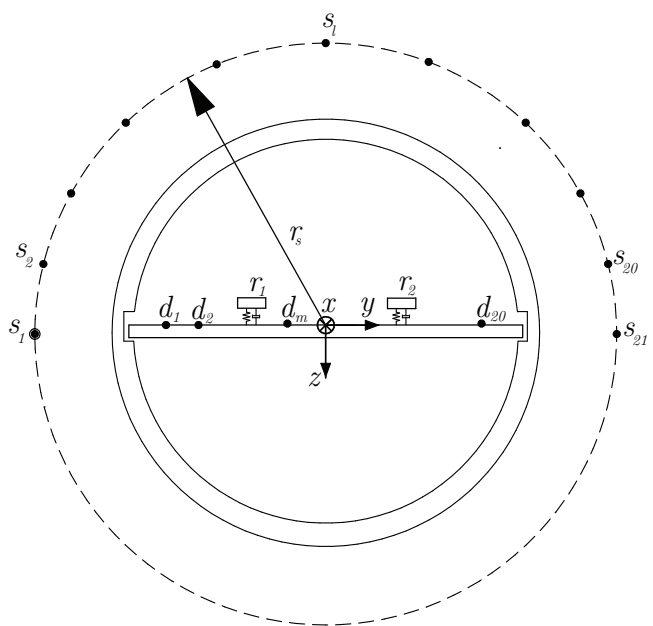

Fig. 7: Geometrical scheme of the receivers located at the soil and at the interior floor.

The Green's functions have been computed for the moving loads with the speeds of $v_{t}=20$ and $25 \mathrm{~m} \mathrm{~s}^{-1}$, which represent typical and maximum train speeds for underground urban railways, respectively. The wavenumber-frequency sampling is developed assuming a maximum frequency of interest, seen from a fixed frame of reference, of $\omega_{\max }=2 \pi 80 \mathrm{rad} / \mathrm{s}$. To compute the corresponding maximum frequency of interest seen from the perspective of the moving frame of reference $\tilde{\omega}_{\max }$, the expression

$$
\tilde{\omega}_{\max }=\omega_{\max }\left(1+\frac{v_{t}}{\beta}\right)
$$

from [44] is used here, where $\beta$ is the speed of S-waves in the soil.

It is proposed to computationally solve Eqs. (8) or (9) by using an inverse fast Fourier Transform (ifft) routine. Consider that the train response is computed at $x=0$. Taking into account that the moving frame of reference is defined as $\tilde{x}=x-v_{t} t$, one can define the sampling vectors for $\tilde{x}$ and $t$ in the basis of the fft as

$$
\tilde{x}_{n}=-\Delta \tilde{x}\left[\begin{array}{lllll}
-N / 2 & \ldots & n-1-N / 2 & \ldots & N / 2-1
\end{array}\right]
$$


and

$$
t_{n}=\Delta t\left[\begin{array}{lllll}
-N / 2 & \ldots & n-1-N / 2 & \ldots & N / 2-1
\end{array}\right],
$$

respectively, where $\Delta \tilde{x}=v_{t} \Delta t$. Thus, the corresponding sampling for $k_{x}$ and $\tilde{\omega}$ should be

$$
k_{x n}=-\Delta k_{x}\left[\begin{array}{lllll}
-N / 2 & \ldots & n-1-N / 2 & \ldots & N / 2-1
\end{array}\right],
$$

and

$$
\tilde{\omega}_{n}=\Delta \tilde{\omega}\left[\begin{array}{lllll}
-N / 2 & \ldots & n-1-N / 2 & \ldots & N / 2-1
\end{array}\right],
$$

respectively, where $\Delta \tilde{\omega}=v_{t} \Delta k_{x}$. Applying a $2 \mathrm{D}$ ifft over this sampling strategy, the diagonal of the resulting $2 \mathrm{D}$ matrix for the specific receiver contains the time response at $x=0$.

The assumption for which the train response is computed at $x=0$ comes from the fact that, in pure 2.5D models, the time response at any arbitrary $x$ is always the same as the one computed at $x=0$ but delayed in time. However, when the DVAs are coupled to the system, the resulting model is no longer longitudinally invariant since it includes a periodical system, which induces a periodical behavior on the time response. Therefore, $x=0$ represents only one of the possible time responses existing within a periodicity. However, the soil response is not significantly affected by this periodical behaviour due to the distance between the track and the receivers in comparison with the longitudinal distance between DVAs. Thus, $x=0$ is taken as the representative time signal for the train response.

\subsection{Train pass-by response}

In this study, the unevenness profiles of the two rails are held to be uncorrelated. As shown by Ntotsios et al. [46], unevenness spectral content of wavelengths shorter than $3 \mathrm{~m}$ should be considered uncorrelated. The train speeds studied in this paper imply that for frequencies larger than $\approx 8 \mathrm{~Hz}$ (most of the frequency range of interest for railway-induced vibrations), the unevenness 
profiles of the two rails are deemed to be uncorrelated. They are modeled using a stochastic random process that is characterized by its power spectral density (PSD) [51] which depends on the rail quality. According to the Federal Railroad Administration (FRA), the unevenness of the rails can be grouped into six classes depending on the rail quality. Class 3 track is used in here.

Fig. 8 shows the time histories of the vertical rail velocity at $x=0$ of the left rail, in the absence of DVAs, due to the train passage at speeds of 20 and $25 \mathrm{~m} \mathrm{~s}^{-1}$. The passage of the train, which has a total of eight axles, can be seen through the eight peaks appearing in the figure. It is apparent that the vertical rail velocity of the rail grows by increasing the train speed. Furthermore, considering the velocity and the length of the train, the time it takes for the train to pass corresponds to the distance between the peaks in time.
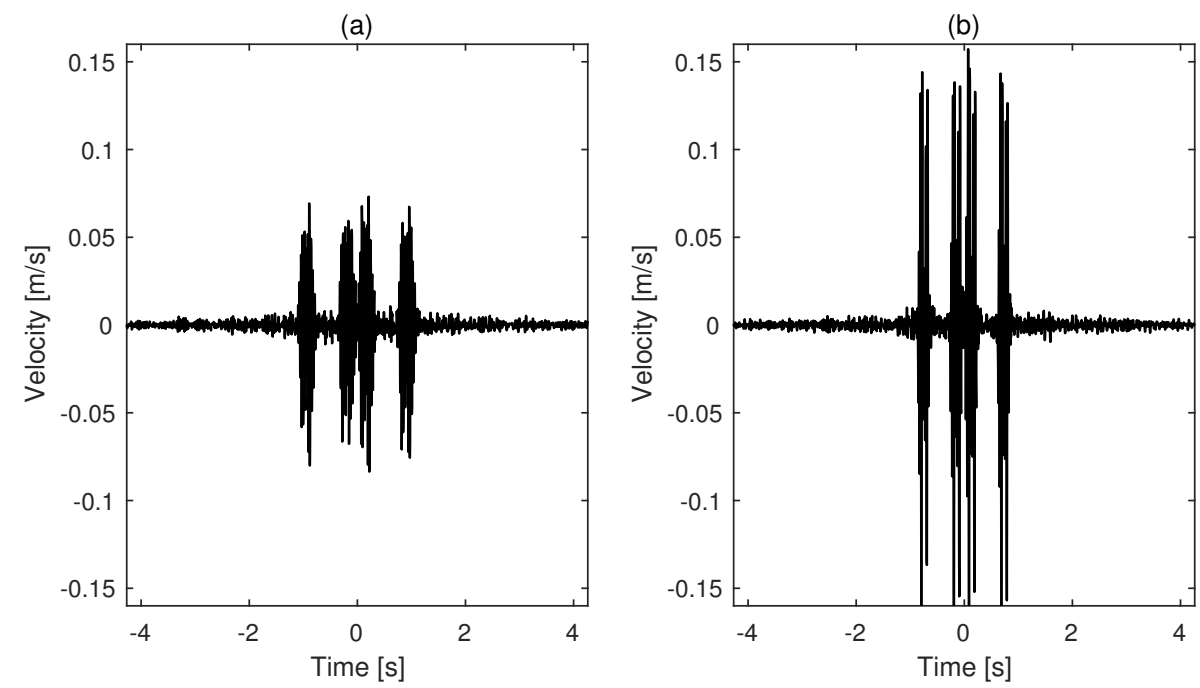

Fig. 8: Time history of the vertical velocity of the left rail due to the train passing at speeds of (a) $v_{t}=20 \mathrm{~m} \mathrm{~s}^{-1}$ and (b) $v_{t}=25 \mathrm{~m} \mathrm{~s}^{-1}$.

Fig. 9 shows the time history of the radial velocity of the hard soil at the receiver $s_{10}$, i.e. located at $\theta=\pi / 2$ and $r_{s}=12 \mathrm{~m}$, due to train passing at speeds of 20 and $25 \mathrm{~m} \mathrm{~s}^{-1}$. In this case, due to the distance between the receiver and the track, the passage of the train axles cannot be clearly identified as compared with the rail response. 

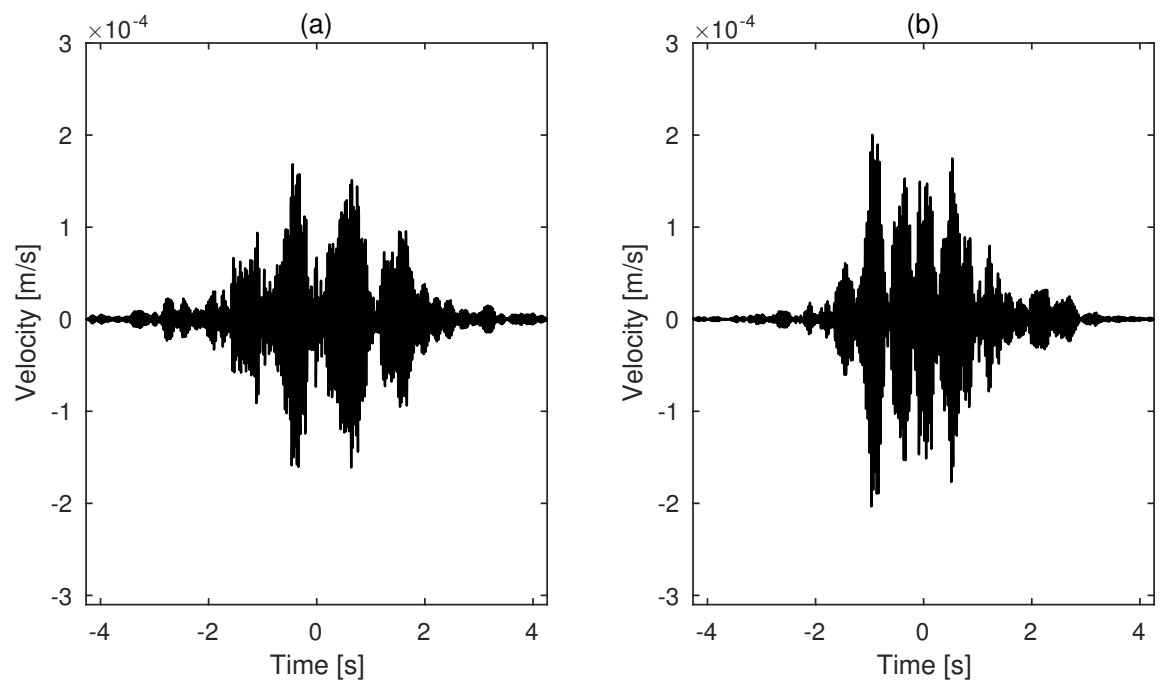

Fig. 9: Time history of the radial velocity of the soil at $\theta=\pi / 2$ and $r_{s}=12 \mathrm{~m}$ due to the train passing at speeds of (a) $v_{t}=20 \mathrm{~m} \mathrm{~s}^{-1}$ and (b) $v_{t}=25 \mathrm{~m} \mathrm{~s}^{-1}$.

Fig. 10 shows the frequency spectrum of the radial velocity for the hard and soft soil at the receiver $s_{10}$, located at $\theta=\pi / 2 \mathrm{rad}$ and $r_{s}=12 \mathrm{~m}$, due to the passage of the train at the speeds of 20 and $25 \mathrm{~m} \mathrm{~s}^{-1}$. It can be observed that the dominant spectral content is in a narrow frequency band for the four cases. Observing the same behavior at the other receivers implies that DVAs would be suitable vibration isolation measures. However, they would be less efficient for the soft soil cases as the dominant frequency band is wider.

\subsection{Optimum parameters of DVAs}

Only one distribution of DVAs is considered in this study. Moreover, the distance between any two consecutive DVAs in a distribution is assumed to be the same. The DVAs in a distribution are considered to have the same mass. Its value, together with the minimum distance between the DVAs and the number of them, are defined in the pre-design stage (common practice in designing DVAs [52]) by ensuring that the static tensions to which the interior floor is subjected would stay approximately unchanged after adding DVAs. This bound is applied in order to avoid structural integrity problems. Thus, the transverse position of DVAs distribution at the interior floor, the distance between two consecutive 

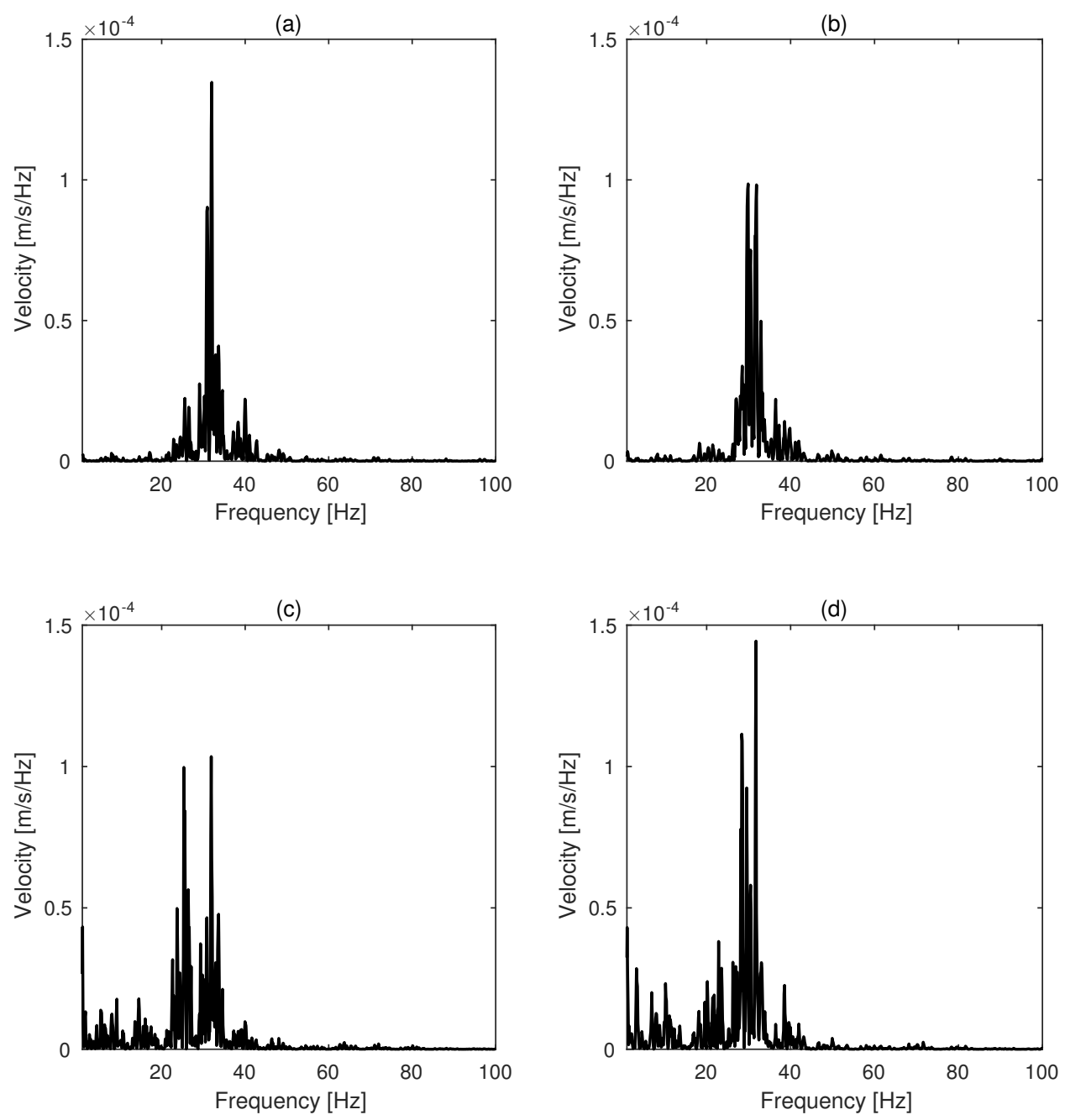

Fig. 10: Frequency spectrum of the radial velocity for the hard soil (top) and soft soil (bottom) at $\theta=\pi / 2 \mathrm{rad}$ and $r_{s}=12 \mathrm{~m}$ due to the train passing at speeds of $(\mathrm{a}, \mathrm{c}) v_{t}=20 \mathrm{~m} \mathrm{~s}^{-1}$ and (b,d) $v_{t}=25 \mathrm{~m} \mathrm{~s}^{-1}$.

DVAs, the natural frequency of the DVAs and viscous damping of the DVAs are defined as design variables in the optimization process.

In this study, the Matlab Global Optimization Toolbox [53] is employed. This toolbox provides functions for finding parameters which minimize an objective while satisfying a set of constraints. The optimization problem set up includes choosing a solver, defining the objective functions and constraints, defin- 
ing optimization options and including parallel processing. In this study, ga (genetic algorithm of MATLAB) has been used as the solver, and the energy flow radiated upwards in the presence of the DVAs has been defined as the objective function. Among bound constraints and linear/non-linear constraints, only the former has been considered. These constraints limit the range from which the design variables can be chosen in the optimization problem. In the ga solver, a set of options can be specified to obtain data from the algorithm while it is running, to drive a random selection of the possible candidates for the solution and to define conditions to terminate the optimization process. In this study, the approach which makes the selection of the candidates being more random than driven has been employed. The maximum number of iterations for the algorithm to perform is used as a condition to terminate the optimization process and it has been defined as triple that of the default value proposed by the algorithm, which is 50. In short, as a general work flow of this algorithm, ga searches for the optimal values of DVAs parameters that can minimize the energy flow radiated upwards from the cylindrical strip due to the passage of the train by considering the bounds constraints. The upper and lower bounds of the design variables and the value of the parameters defined in the pre-design stage are given in the following:

1. Number of DVAs distributions $M$ : Only one longitudinal distribution of DVAs is considered.

2. Transverse position of DVAs distribution $y_{d}$. It can be chosen from 20 possible positions defined previously in Subsection 5.2.

3. Distance between two consecutive DVAs $l_{d}$ : It is defined as a discrete variable, which can be chosen from $1 \mathrm{~m}$ to $8 \mathrm{~m}$ as the lower and upper bounds of this design variable, respectively, with a space step of $0.5 \mathrm{~m}$. The space step has been restricted to $0.5 \mathrm{~m}$ because of the size of the DVAs to be used.

4. The mass of the DVAs $m_{d}$ : It is defined in the pre-design stage. All DVAs 
in a distribution are assumed to have the same mass of $800 \mathrm{~kg}$.

5. Number of DVAs in a distribution $N$ : It is also defined in the pre-design stage, taking a value of 15 DVAs.

6. The natural frequency of the DVAs $f_{d}$ : It is defined as a discrete variable that can be chosen from the values of the fixed frame frequency (i.e. seen from the fixed frame of reference) given by the wavenumber-frequency sampling defined in Subsection 5.2.

7. The viscous damping of the DVAs $c_{d}$ : It is defined as a discrete variable with lower and upper bounds of $5 \mathrm{kN} \mathrm{s} \mathrm{m}^{-1}$ and $500 \mathrm{kN} \mathrm{s} \mathrm{m}^{-1}$, respectively. It is considered to be a total amount of 316 possible values linearly distributed between the defined bounds.

An optimization process based on GA has been carried out to minimize the energy flow radiated upwards due to the application of a distribution of DVAs. The following four cases have been studied: Case H25: hard soil and train speed of $v_{t}=25 \mathrm{~m} \mathrm{~s}^{-1}$; Case H20: hard soil and train speed of $v_{t}=20 \mathrm{~m} \mathrm{~s}^{-1}$; Case S20: soft soil and train speed of $v_{t}=20 \mathrm{~m} \mathrm{~s}^{-1}$ and Case S25: soft soil and train speed of $v_{t}=25 \mathrm{~m} \mathrm{~s}^{-1}$. The resulting optimum values of the DVAs parameters and the associated insertion loss (IL) on the radiated energy flow are presented in Table 7. The IL was computed as

$$
\mathrm{IL}=10 \log _{10}\left(\frac{E}{E^{\prime}}\right)
$$

where $E$ and $E^{\prime}$ represent the radiated energy without and with the application of the DVAs. 
Table 7: The optimum values of DVAs parameters and resulting IL.

\begin{tabular}{cccccc}
\hline Case & $y_{d}(\mathrm{~m})$ & $l_{d}(\mathrm{~m})$ & $f_{d}(\mathrm{~Hz})$ & $c_{d}\left(\mathrm{kN} \mathrm{s} \mathrm{m}^{-1}\right)$ & $\mathrm{IL}(\mathrm{dB})$ \\
\hline $\mathrm{H} 20$ & 3.55 & 7.5 & 31.52 & 14.09 & 6.2 \\
$\mathrm{H} 25$ & -2.45 & 4.5 & 28.83 & 27.07 & 6.6 \\
$\mathrm{~S} 20$ & 1.05 & 7 & 31.17 & 20.65 & 5.3 \\
$\mathrm{~S} 25$ & -3.45 & 6 & 31.6 & 27.76 & 5.8 \\
\hline
\end{tabular}

Fig. 11 shows the mean power flow radiated upwards from the cylindrical strip with and without DVAs in the time domain for the four studied cases. The total radiated energy with and without DVAs is also given for each case. This mean power flow has been computed using the velocities and tractions over the cylindrical strip at $x_{e}=0$, which is defined in accordance with the space-time sampling previously defined in Subsection 5.2. The expression to compute the mean power flow can be derived from Eq. (31) and it is

$$
P(t)=r_{m} \Delta x \int_{\theta_{1}}^{\theta_{2}} \mathbf{v}\left(x_{e}, \theta, t\right) \cdot \boldsymbol{\tau}\left(x_{e}, \theta, t\right) \mathrm{d} \theta .
$$

As generally expected, the radiated energy increases for both soft and hard soil when the speed of the train increases. The comparison of the radiated power flow or the total radiated energy with and without the application of DVAs indicates that using DVAs results in a notable decrease in radiated power flow or total radiated energy for all studied cases. For all the studied cases, Fig. 11 shows that the mean power flow becomes negative-valued at specific instants of time. This behavior of the mean power flow radiated upwards by a double-deck tunnel was previously observed by Clot [54] for the case of a quasi-static point load. A meaningful explanation of this phenomenon is that the elastic surface waves that travel along the tunnel cavity exhibit a particle motion very similar to the one presented by Rayleigh surface waves [55]. This close resemblance indicates the existence of elliptical particle motion of the soil close to the tunnel structure. This means that at some time intervals there are particle motions of the soil toward the tunnel rather than away from it. This 

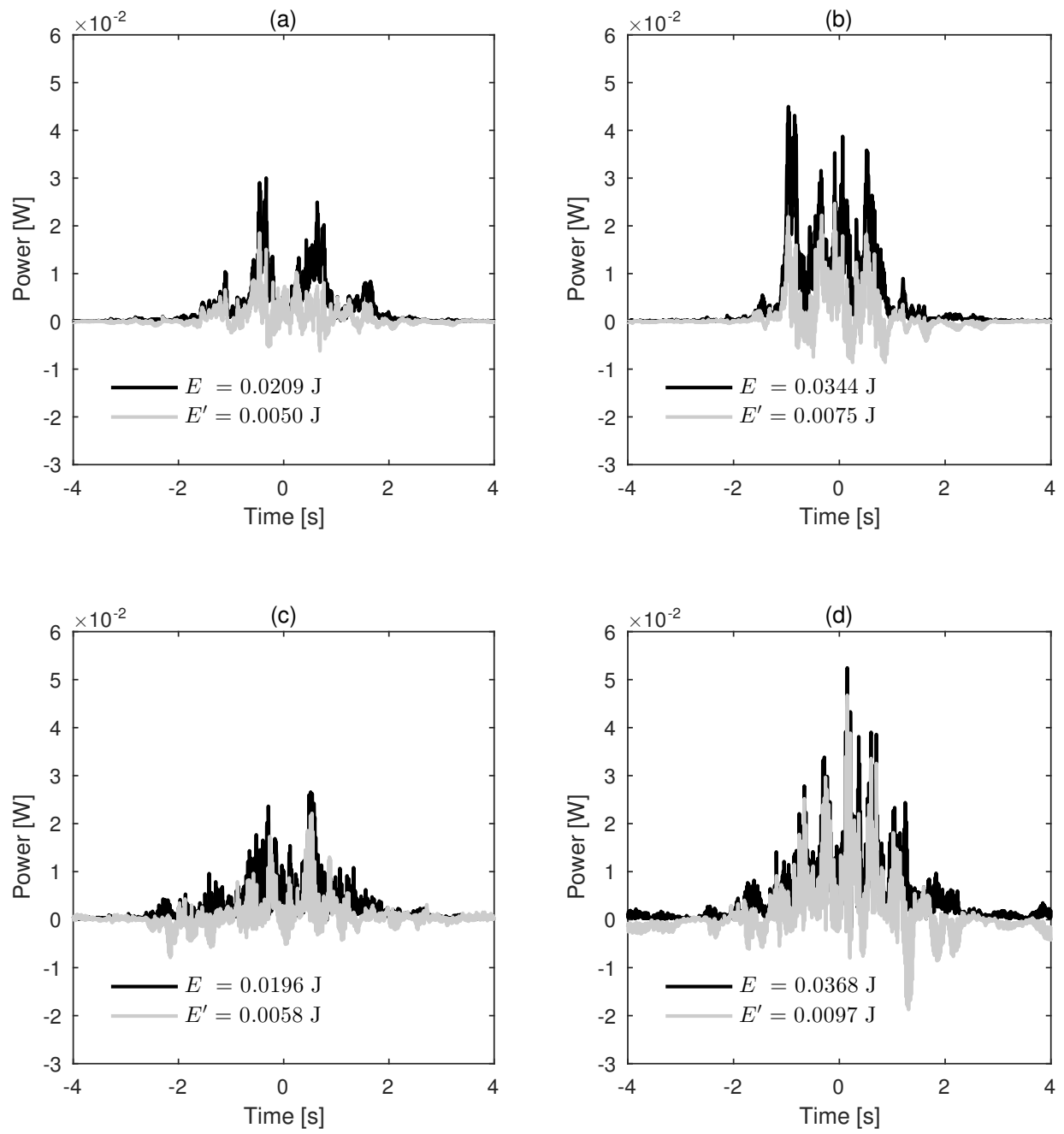

Fig. 11: Mean power flow radiated upwards over the cylindrical strip in the time domain for the cases (a) H20, (b) H25, (c) S20 and (d) S25. The grey and black lines represent the results with and without DVAs, respectively. The total radiated energy is presented for each case.

results in negative radiated vibration power flow if the positive power flow is defined as the power radiated away from the tunnel.

Fig. 12 shows the energy spectral density (ESD) of the previously computed mean power flow, with and without DVAs, for the four studied cases. As expected, the results of computing the total radiated energy using ESD is the same value previously achieved from the mean power flow in the time domain. 

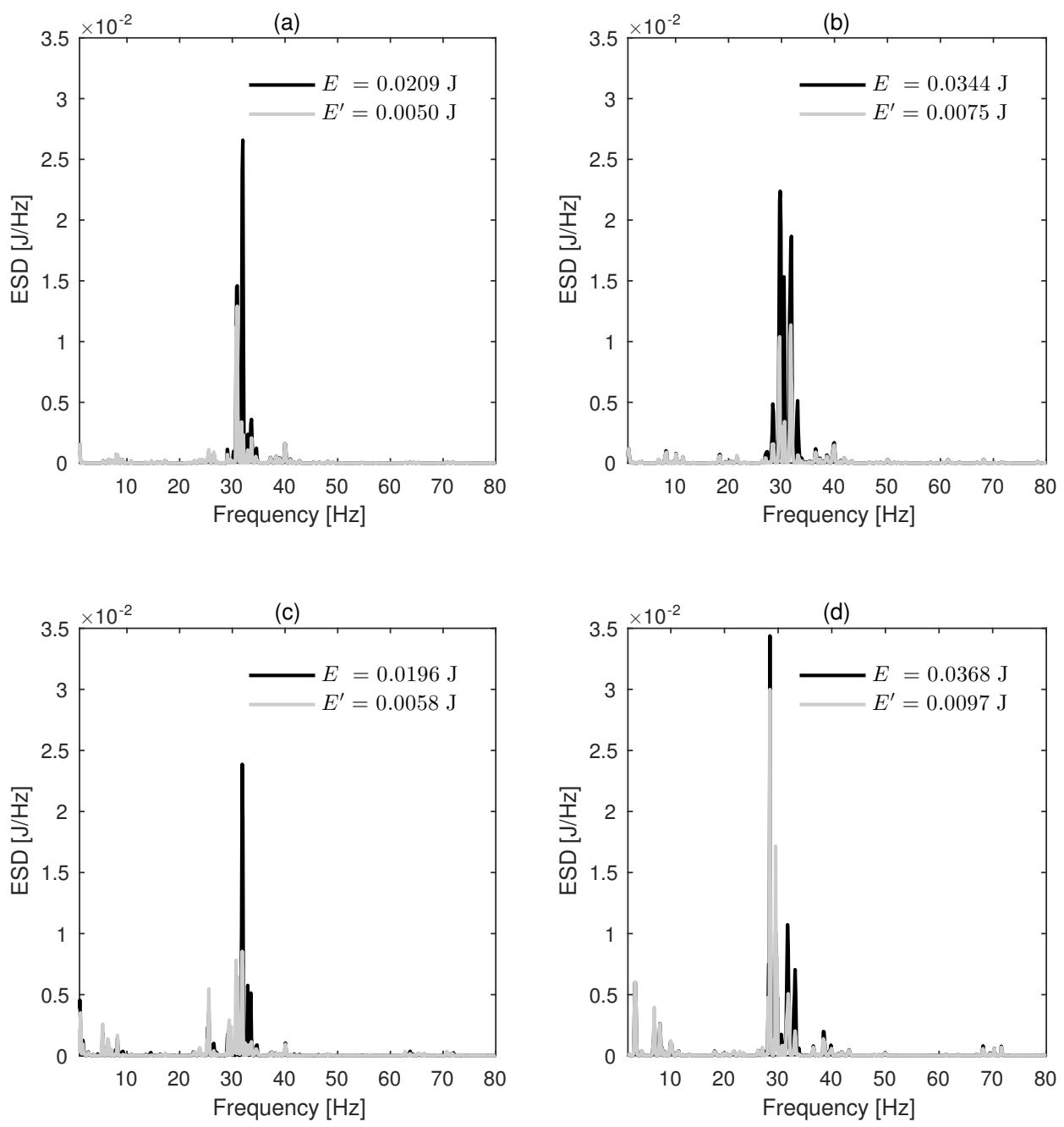

Fig. 12: ESD for cases (a) H20, (b) H25, (c) S20 and (d) S25. The grey and black lines represent the results with and without DVAs, respectively. The total radiated energy is also presented for each case.

It can be observed in Fig. 12 that the most significant energy content is concentrated in a frequency range between 25 to $35 \mathrm{~Hz}$. It is noteworthy that the optimized natural frequencies of DVAs have been obtained in this range of frequency, which makes them effective in minimizing the radiated energy. The range of frequency in which most of the energy content is found and at which the DVAs are effective can be seen more clearly in Fig. 13, which represents 
energy spectrum in one-third octave band for the considered cases with and without application of DVAs. The presented octave bands are normalized with the length of the time signal, which is 8.54 seconds.
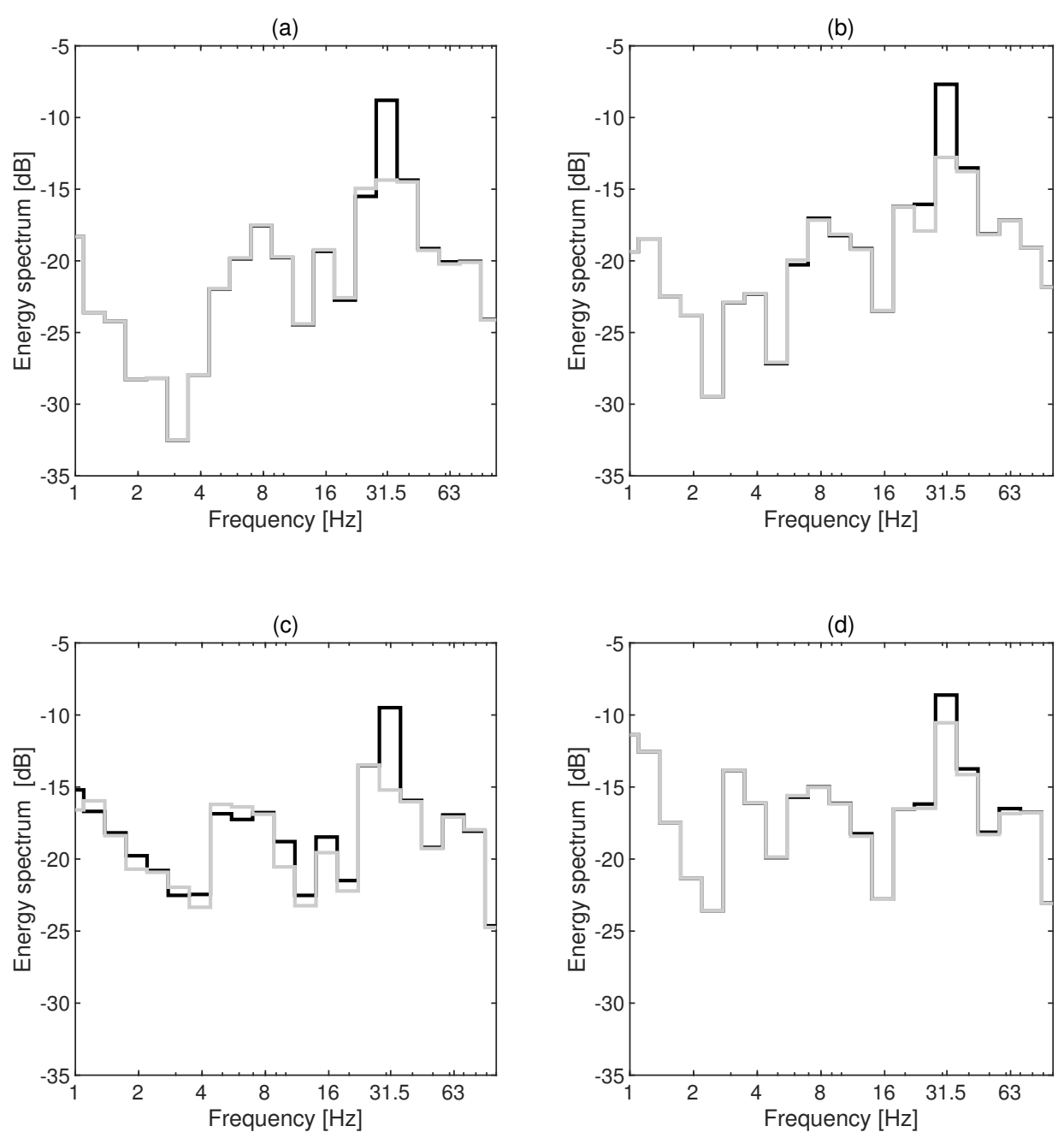

Fig. 13: One-third octave bands spectrum of energy in dB (dB reference $1 \mathrm{~J}$ ) for cases (a) H20, (b) H25, (c) S20 and (d) S25. The grey and black lines represent the results with and without DVAs, respectively.

In order to study the radiation pattern of the energy flow, the energy radiated through the cylindrical strip has been computed as a function of $\theta$, for all studied 
cases and with and without the application of DVAs, using

$$
E(\theta)=r_{m} \Delta \mathrm{x} \int_{-\infty}^{+\infty} \mathbf{v}(0, \theta, t) \cdot \boldsymbol{\tau}(0, \theta, t) \mathrm{d} t
$$

The results are shown in Fig. 14. One thing to note is that depending on the type of the soil, the energy flow radiation pattern would differ. For hard soil cases, the energy mostly radiates over the center of the strip, however, for the soft soil cases, it radiates mostly over the sides of the strip. For both cases, DVAs are significantly affecting the $\theta$ distribution of the radiation pattern. This is because the mode shapes of the interior floor, which are modified by the application of the DVAs, are strongly related with the radiation pattern distribution, as discussed previously by Clot et al. [49] in a 2D power flow analysis of the double-deck tunnel subjected to a harmonic line load.
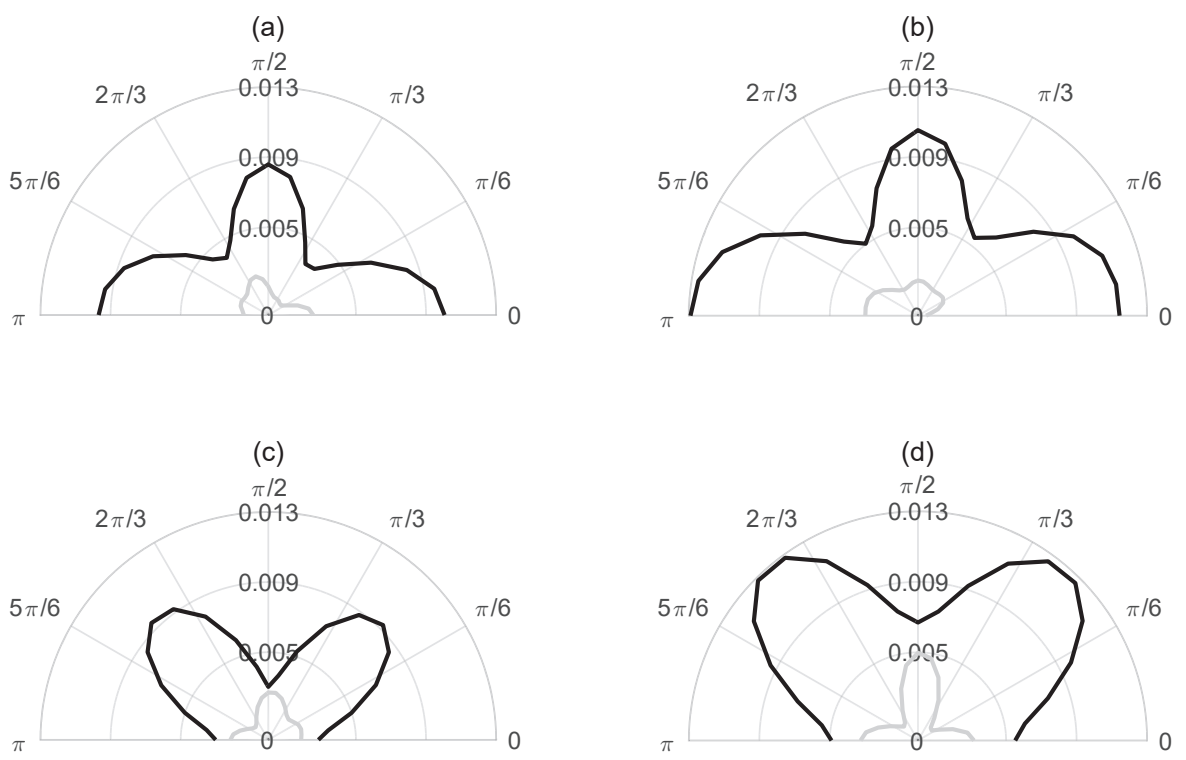

Fig. 14: Energy radiated over cylindrical strip in $\mathrm{J}$ as a function of $\theta$ for cases (a) H20, (b) H25, (c) S20 and (d) S25. The grey and black lines represent the results with and without DVAs, respectively.

In order to investigate the relation between the natural frequency of the optimal DVAs and the dynamical behavior of the original system, two figures 
are presented. On the one hand, Fig. 15 shows the radial velocity Green's functions of the hard soil case at three receivers located at $\theta=0, \theta=\pi / 2$ and $\theta=\pi \mathrm{rad}$, and at a radius of $12 \mathrm{~m}$ due to the force applied on the right rail. The red areas show local maximums of the velocity Green's functions and they represent an approximation to the dispersion curves of the system. From this approximation, three propagation modes of the interior floor coupled to the tunnel-soil system can be observed: the first and the third are antisymmetric (not observed at $\theta=\pi / 2$ ) and the second is symmetric (the only one appearing at $\theta=\pi / 2$ ). The inclined dashed black lines plotted in Fig. 15 represent combinations of $k_{x}$ and $\omega$ of constant $\tilde{\omega}$ for the specific speed of $25 \mathrm{~m} \mathrm{~s}^{-1}$. On the other hand, Fig. 16 shows dynamic wheel-rail interaction forces for the same case study but considering $20 \mathrm{~m} \mathrm{~s}^{-1}$ and $25 \mathrm{~m} \mathrm{~s}^{-1}$. For both figures, the computations have been done without considering coupled DVAs. Comparing these two figures with the results shown in Table 7, where the natural frequency of the optimal DVAs for the hard soil case is $31.52 \mathrm{~Hz}$ for $v_{t}=20 \mathrm{~m} \mathrm{~s}^{-1}$ and $28.83 \mathrm{~Hz}$ for $v_{t}=25 \mathrm{~m} \mathrm{~s}^{-1}$, one can observe that the DVAs are targeting the second propagation mode of the track-interior floor-tunnel-soil system. This can be deduced because of two reasons: firstly, the inclined black lines of constant $\tilde{\omega}$ have a slope far from the tangents to the dispersion curves except for wavenumbers close to zero, resulting in that the frequency associated to these propagation modes is mostly the one of the $2 \mathrm{D}$ problem; secondly, the contact forces have a significant amount of spectral energy close to the resonance frequency associated to the second propagation mode for the $2 \mathrm{D}$ case. 
(a)

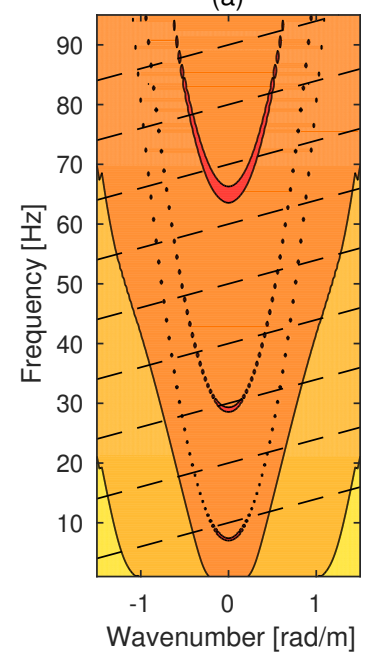

(b)

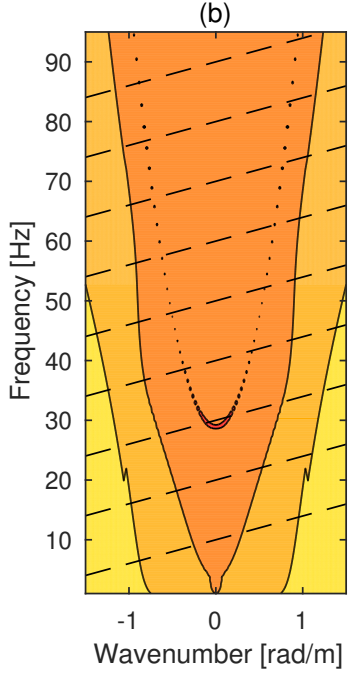

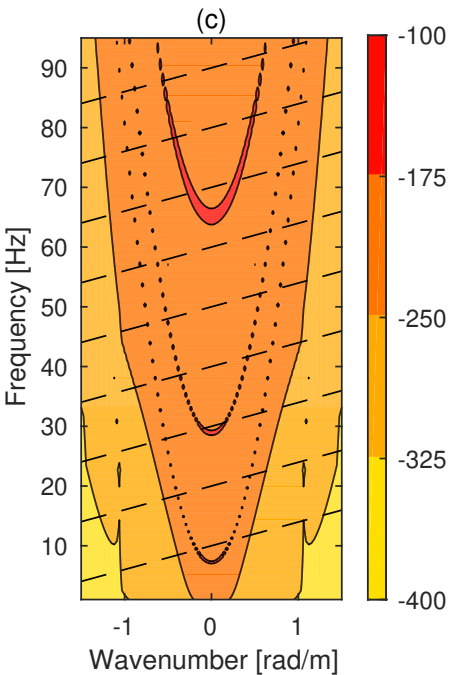

Fig. 15: The radial velocity Green's functions of the hard soil in $\mathrm{dB}\left(\mathrm{dB}\right.$ reference $\left.1 \mathrm{~m} \mathrm{~N}^{-1} \mathrm{~s}^{-1}\right)$ at the receivers located at $r_{s}=12 \mathrm{~m}$ and (a) $\theta=0$, (b) $\theta=\pi / 2 \mathrm{rad}$ and (c) $\theta=\pi \mathrm{rad}$ for $v_{t}=25 \mathrm{~m} \mathrm{~s}^{-1}$. Inclined dashed black lines denote points of constant $\tilde{\omega}$ for the specific speed of $25 \mathrm{~m} \mathrm{~s}^{-1}$.

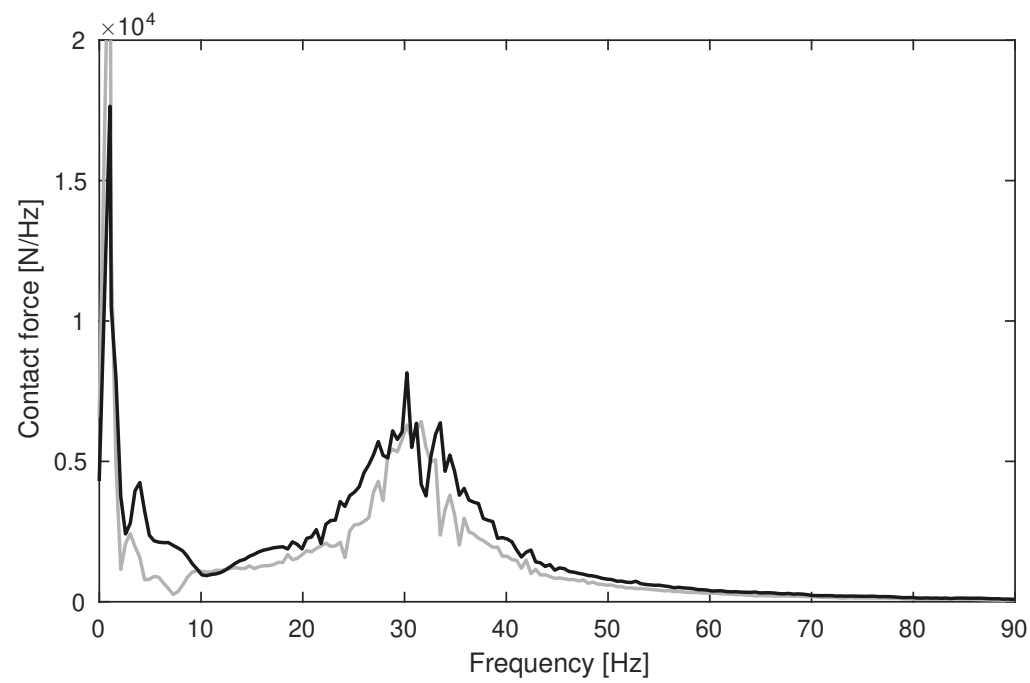

Fig. 16: Contact forces caused by wheel-rail interaction associated to $v_{t}=20 \mathrm{~m} \mathrm{~s}^{-1}$ (grey) and $v_{t}=25 \mathrm{~m} \mathrm{~s}^{-1}$ (black).

The effect of the DVAs on the dynamic response of the rails is also studied. Fig. 17 shows the one-third octave band spectrum of the vertical velocity of 
the left rail with and without DVAs, for two different train speeds. It can be seen from the figures that the application of DVAs on the interior floor of the tunnel has little effect on the dynamic response of the track. Because of that, the train-track dynamic forces can be computed before the optimization process, only once. If the track is already constructed, these forces can be obtained using a hybrid approach [12] that enhance the accuracy of the DVAs efficiency prediction. However, it is important to highlight that this result is only associated to the present problem parameters. In cases where the DVAs natural frequency is similar to the rail/fasteners natural frequency, this conclusion is no longer valid.
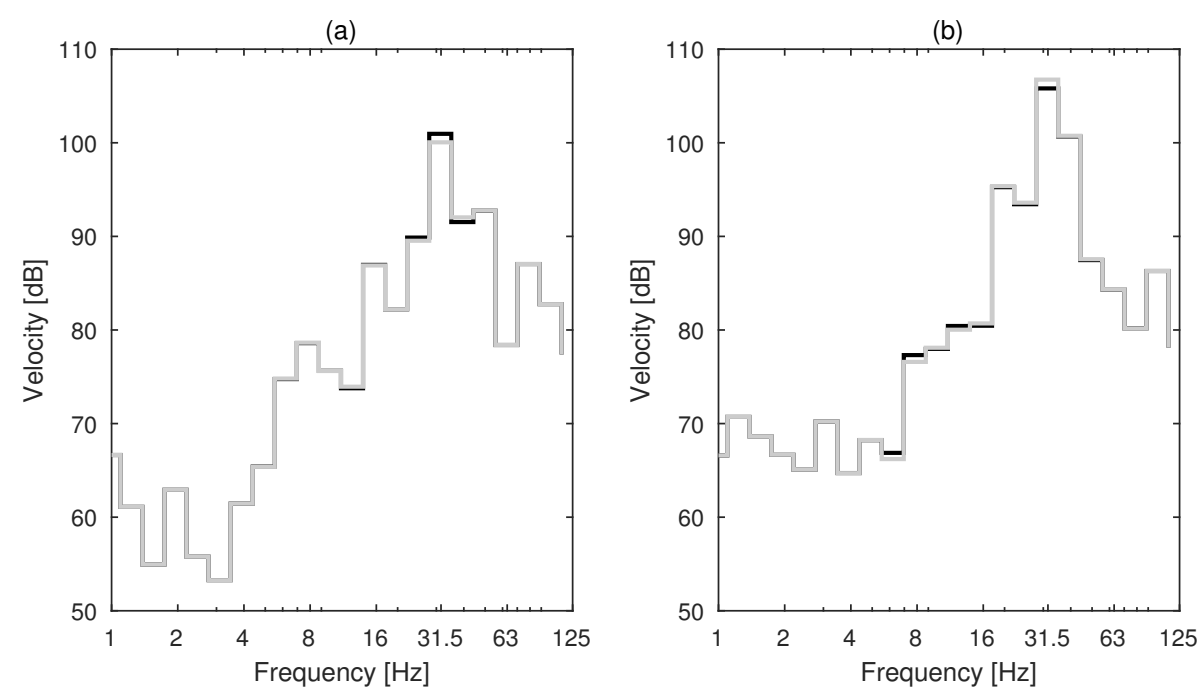

Fig. 17: One-third octave band spectrum of the vertical velocity of the left rail in $\mathrm{dB}$ ( $\mathrm{dB}$ reference $10^{-8} \mathrm{~m} \mathrm{~s}^{-1}$ ) for the train speeds of (a) $v_{t}=20 \mathrm{~m} \mathrm{~s}^{-1}$ and (b) $v_{t}=25 \mathrm{~m} \mathrm{~s}^{-1}$. The grey and black lines represent the results with and without DVAs, respectively.

\section{Conclusions}

The main objective of this paper is to evaluate the efficiency of the application of DVAs in a double-deck tunnel to mitigate the underground railwayinduced ground-borne vibration. The total energy flow radiated upwards by a double-deck tunnel due to train pass-by has been considered to be the criterion 
to assess the performance of DVAs. A semi-analytical model of a double-deck tunnel embedded in a full-space has been used to compute the energy flow radiated upwards by the double-deck tunnel due to the train pass-by over a track system located at its interior floor. A methodology to couple DVAs to any subsystem of a railway infrastructure has been explained and was then employed to couple DVAs to the interior floor of a double-deck tunnel. Taking into account the crucial role of the DVAs parameter in their performance, a global optimization approach based on GA has been used to obtain the optimal parameters of DVAs to minimize the total radiated energy.

The performance of one longitudinal distribution of DVAs has been evaluated for two different types of soil and two different train speeds. In all of the four cases, they have been found to be efficient in reducing the total radiated energy flow by the tunnel. The obtained insertion loss of the total radiated energy flow due to the application of DVAs for the four cases shows that the harder the soil is and the faster the train, the more effective are the optimized DVAs. In the case of the hard soil and the train speed of $25 \mathrm{~m} / \mathrm{s}$, a reduction of $6.6 \mathrm{~dB}$ in the total radiated energy flow has been achieved. The results show that DVAs provide significant vibration attenuation benefits by tuning their optimum natural frequencies to be set down in the range of frequency where most of the energy spectral content is concentrated. Another finding is that the energy flow radiation pattern from the tunnel is strongly modified after the application of the DVAs, as they modify the mode shapes of the interior floor. However, the application of DVAs does not considerably affect the dynamic response of the rails. It is expected that using more than one longitudinal distribution of DVAs would result in a greater reduction in the total radiated energy flow. It can be noted that DVAs would be a more cost-effective solution for existing underground railway networks than, for example, vibration isolation screens or building base isolation, as their implementation would be cheaper than other vibration countermeasures. Noteworthy, it is expected that DVAs as countermeasures for railway-induced ground-borne vibrations would be generally more effective for the cases with a sharper vibration frequency spectra of 
the tunnel response (like the ones in double-deck tunnels) rather than the ones with smooth vibration frequency spectra (like the ones in conventional tunnels). Nevertheless, their effectiveness for conventional tunnels and at-grade railway infrastructures is not studied in detail yet.

\section{Acknowledgments}

This work has been carried out in the context of the Industrial Doctorates Plan, with the financial support of Agència de Gestió d'Ajuts Universitaris i de Recerca (AGAUR) from Generalitat de Catalunya and the company AV Ingenieros. It was developed in a partnership with Universitat Politècnica de Catalunya (UPC). The authors would like to extend their gratitude to the ISIBUR project, Innovative Solutions for the Isolation of Buildings from Underground Railway-induced Vibrations, funded by Ministerio de Economía y Competitivad de España (TRA2014 52718-R). The second author also wants to acknowledge the funds provided by the NVTRail project, Noise and Vibrations induced by railway traffic in tunnels: an integrated approach, with grant reference POCI-01-0145-FEDER-029577, funded by FEDER through COMPETE2020 (Programa Operacional Competitividade e Internacionalização (POCI)) and by national funds (PIDDAC) through FCT/MCTES.

\section{References}

[1] G. Lombaert, G. Degrande, B. Vanhauwere, B. Vandeborght, S. François, The control of ground-borne vibrations from railway traffic by means of continuous floating slabs, Journal of Sound and Vibration 297 (2006) 946961.

[2] P. Alves Costa, R. Calçada, A. Silva Cardoso, Ballast mats for the reduction of railway traffic vibrations. Numerical study, Soil Dynamics and Earthquake Engineering 42 (2012) 137-150. 
[3] J. Talbot, W. Hamad, H. Hunt, Base-isolated buildings and the addedmass effect, in: Proceedings of ISMA 2014-International Conference on Noise and Vibration Engineering and USD 2014-International Conference on Uncertainty in Structural Dynamics, 2014, pp. 943-954.

[4] J. Talbot, H. Hunt, On the performance of base-isolated buildings, Building Acoustics 7 (2000) 163-178.

[5] L. Andersen, S. R. Nielsen, Reduction of ground vibration by means of barriers or soil improvement along a railway track, Soil Dynamics and Earthquake Engineering 25 (2005) 701-716.

[6] P. Coulier, S. François, G. Degrande, G. Lombaert, Subgrade stiffening next to the track as a wave impeding barrier for railway induced vibrations, Soil Dynamics and Earthquake Engineering 48 (2013) 119-131.

[7] X. Sheng, C. J. C. Jones, D. J. Thompson, Prediction of ground vibration from trains using the wavenumber finite and boundary element methods, Journal of Sound and Vibration 293 (2006) 575-586.

[8] S. François, M. Schevenels, P. Galvín, G. Lombaert, G. Degrande, A 2.5D coupled FE-BE methodology for the dynamic interaction between longitudinally invariant structures and a layered halfspace, Computer Methods in Applied Mechanics and Engineering 199 (2010) 1536-1548.

[9] P. Alves Costa, R. Calçada, A. Silva Cardoso, Track-ground vibrations induced by railway traffic: In-situ measurements and validation of a $2.5 \mathrm{D}$ FEM-BEM model, Soil Dynamics and Earthquake Engineering 32 (2012) $111-128$.

[10] P. Amado-Mendes, P. Alves Costa, L. M. C. Godinho, P. Lopes, 2.5D MFSFEM model for the prediction of vibrations due to underground railway traffic, Engineering Structures 104 (2015) 141-154. 
[11] J. C. O. Nielsen, G. Lombaert, S. François, A hybrid model for prediction of ground-borne vibration due to discrete wheel/rail irregularities, Journal of Sound and Vibration 345 (2015) 103-120.

[12] K. A. Kuo, H. Verbraken, G. Degrande, G. Lombaert, Hybrid predictions of railway induced ground vibration using a combination of experimental measurements and numerical modelling, Journal of Sound and Vibration 373 (2016) 263-284.

[13] J. A. Forrest, H. E. M. Hunt, A three-dimensional tunnel model for calculation of train-induced ground vibration, Journal of Sound and Vibration 294 (2006) 678-705.

[14] J. A. Forrest, H. E. M. Hunt, Ground vibration generated by trains in underground tunnels, Journal of Sound and Vibration 294 (2006) 706-736.

[15] M. Hussein, S. François, M. Schevenels, H. Hunt, J. Talbot, G. Degrande, The fictitious force method for efficient calculation of vibration from a tunnel embedded in a multi-layered half-space, Journal of Sound and Vibration 333 (2014) 6996-7018.

[16] A. Tadeu, E. Kausel, Green's functions for two-and-a-half-dimensional elastodynamic problems, Journal of Engineering Mechanics - ASCE 126 (2000) 1093-1097.

[17] B. Noori, R. Arcos, J. Romeu, A. Clot, A new method based on 3D stiffness matrices in Cartesian coordinates for computation of 2.5D elastodynamic Green's functions of layered half-spaces, Soil Dynamics and Earthquake Engineering 89 (2018) 154-158.

[18] J. Q. Sun, M. R. Jolly, M. A. Norris, Passive, Adaptive and Active Tuned Vibration Absorbers-A Survey, Journal of Mechanical Design 117 (1995) 234-242.

[19] L. Kela, P. Vähäoja, Recent studies of adaptive tuned vibration absorbers/neutralizers, Applied Mechanics Reviews 62 (2009) 1-9. 
[20] I. Kourakis, Structural systems and tuned mass dampers of super-tall buildings : case study of Taipei 101, Ph.D. thesis, Massachusetts Institute of Technology, 2007.

[21] D. E. Newland, Vibration of the London Millennium Bridge: cause and cure, International Journal of Acoustics and Vibration 8 (2003) 9-14.

[22] P. Nawrotzki, Tuned-mass systems for the dynamic upgrade of buildings and other structures, in: Eleventh East Asia-Pacific Conference on Structural Engineering \& Construction (EASEC-11) Building a Sustainable Environment, Taipei Taiwan, Citeseer, 2008.

[23] P. Watts, On a method of reducing the rolling of ships at sea, Transactions of the Institution of Naval Architects 24 (1883) 165-190.

[24] H. Frahm, Device for damping vibration of bodies, U.S. Patent No. 989958 (1911).

[25] J. Ormondroyd, The theory of the dynamic vibration absorber, trans. asme, Transactions of the American Society of Mechanical Engineers, Applied Mechanics Division 50 (1928).

[26] M. Zilletti, S. J. Elliott, E. Rustighi, Optimisation of dynamic vibration absorbers to minimise kinetic energy and maximise internal power dissipation, Journal of sound and vibration 331 (2012) 4093-4100.

[27] T. Asami, O. Nishihara, A. M. Baz, Analytical solutions to $\mathrm{H}_{\infty}$ and $\mathrm{H}_{2}$ optimization of dynamic vibration absorbers attached to damped linear systems, Journal of vibration and acoustics 124 (2002) 284-295.

[28] M. N. Hadi, Y. Arfiadi, Optimum design of absorber for MDOF structures, Journal of Structural Engineering 124 (1998) 1272-1280.

[29] B. Noori, A. Farshidianfar, Optimum design of dynamic vibration absorbers for a beam, based on $\mathrm{H}_{\infty}$ and $\mathrm{H}_{2}$ optimization, Archive of Applied Mechanics 83 (2013) 1773-1787. 
[30] M. P. Singh, S. Singh, L. M. Moreschi, Tuned mass dampers for response control of torsional buildings, Earthquake Engineering \& Structural Dynamics 31 (2002) 749-769.

[31] N. B. Desu, S. Deb, A. Dutta, Coupled tuned mass dampers for control of coupled vibrations in asymmetric buildings, Structural Control and Health Monitoring 13 (2006) 897-916.

[32] Y. Arfiadi, M. Hadi, Optimum placement and properties of tuned mass dampers using hybrid genetic algorithms, International Journal of Optimization in Civil Engineering 1 (2011) 167-187.

[33] M. Mohebbi, K. Shakeri, Y. Ghanbarpour, H. Majzoub, Designing optimal multiple tuned mass dampers using genetic algorithms (GAs) for mitigating the seismic response of structures, Journal of Vibration and Control 19 (2013) 605-625.

[34] S. Zhu, J. Yang, H. Yan, L. Zhang, C. Cai, Low-frequency vibration control of floating slab tracks using dynamic vibration absorbers, Vehicle System Dynamics 53 (2015) 1296-1314.

[35] J. P. Den Hartog, Mechanical vibrations, Courier Corporation, 1985.

[36] Q. Wang, J. Zeng, L. Wei, C. Zhou, B. Zhu, Reduction of vertical abnormal vibration in carbodies of low-floor railway trains by using a dynamic vibration absorber, Proceedings of the Institution of Mechanical Engineers, Part F: Journal of Rail and Rapid Transit 232 (2018) 1437-1447.

[37] D. Thompson, C. Jones, T. Waters, D. Farrington, A tuned damping device for reducing noise from railway track, Applied Acoustics 68 (2007) 43-57.

[38] T. Wu, Effects on short pitch rail corrugation growth of a rail vibration absorber/damper, Wear 271 (2011) 339-348.

[39] W. Ho, B. Wong, D. England, Tuned mass damper for rail noise control, in: Noise and Vibration Mitigation for Rail Transportation Systems, Springer, 2012, pp. 89-96. 
[40] W. Ho, B. Wong, D. Tsui, C. Kong, Reducing rail corrugation growth by tuned mass damper, in: Proceedings of the 11th International Workshop of Railway Noise, 2013.

[41] A. Clot, R. Arcos, J. Romeu, B. Noori, Prediction of the isolation efficiency of vibration countermeasures for a double-deck tunnel, in: Proceedings of EuroRegio 2016, 2016.

[42] A. Clot, R. Arcos, B. Noori, J. Romeu, Isolation of vibrations induced by railway traffic in double-deck tunnels using elastomeric mats, in: 24th International Congress on Sound and Vibration, ICSV 2017, 2017.

[43] A. Clot, R. Arcos, J. Romeu, T. Pàmies, Dynamic response of a doubledeck circular tunnel embedded in a full-space, Tunnelling and Underground Space Technology 59 (2016) 146-156.

[44] G. Lombaert, G. Degrande, Ground-borne vibration due to static and dynamic axle loads of InterCity and high-speed trains, Journal of Sound and Vibration 319 (2009) 1036-1066.

[45] M. Heckl, G. Hauck, R. Wettschureck, Structure-borne sound and vibration from rail traffic, Journal of Sound and Vibration 193 (1996) 175-184.

[46] E. Ntotsios, D. Thompson, M. Hussein, The effect of track load correlation on ground-borne vibration from railways, Journal of Sound and Vibration 402 (2017) 142-163.

[47] X. Lei, N. A. Noda, Analyses of dynamic response of vehicle and track coupling system with random irregularity of track vertical profile, Journal of Sound and Vibration 258 (2002) 147-165.

[48] M. F. M. Hussein, H. E. M. Hunt, A power flow method for evaluating vibration from underground railways, Journal of Sound and Vibration 293 (2006) 667-679. 
[49] A. Clot, J. Romeu, R. Arcos, S. R. Martín, A power flow analysis of a double-deck circular tunnel embedded in a full-space, Soil Dynamics and Earthquake Engineering 57 (2014) 1-9.

[50] A. Clot, J. Romeu, R. Arcos, An energy flow study of a double-deck tunnel under quasi-static and harmonic excitations, Soil Dynamics and Earthquake Engineering 89 (2016) 1-4.

[51] S. Gupta, W. F. Liu, G. Degrande, G. Lombaert, W. N. Liu, Prediction of vibrations induced by underground railway traffic in Beijing, Journal of Sound and Vibration 310 (2008) 608-630.

[52] G. C. Marano, R. Greco, B. Chiaia, A comparison between different optimization criteria for tuned mass dampers design, Journal of Sound and Vibration 329 (2010) 4880-4890.

[53] MATLAB, version 9.2.0 (R2017a), The MathWorks Inc., Natick, Massachusetts, 2017.

[54] A. Clot, A dynamical model of a double-deck circular tunnel embedded in a full-space, Ph.D. thesis, Universitat Politècnica de Catalunya, 2014.

[55] A. Boström, A. D. Burden, Propagation of elastic surface waves along a cylindrical cavity and their excitation by a point force, Journal of the Acoustical Society of America 72 (1982) 998-1004. 